\title{
Mapping luminous blue compact galaxies with VIRUS-P
}

\section{Morphology, line ratios, and kinematics}

\author{
L. M. Cairós ${ }^{1}$, N. Caon ${ }^{2,3}$, B. García Lorenzo ${ }^{2,3}$, A. Kelz ${ }^{1}$, M. Roth ${ }^{1}$, P. Papaderos ${ }^{4}$, and O. Streicher ${ }^{1}$ \\ ${ }^{1}$ Leibniz-Institut für Astrophysik, An der Sternwarte 16, 14482 Potsdam, Germany \\ e-mail: [luzma; akelz;mmroth;ole]@aip.de \\ 2 Instituto de Astrofísica de Canarias, 38200 La Laguna, Tenerife, Spain \\ 3 Departamento de Astrofísica, Universidad de la Laguna, 38206 La Laguna, Tenerife, Spain \\ e-mail: [nicola.caon; bgarcia]@iac.es \\ ${ }^{4}$ Centro de Astrofísica and Faculdade de Ciências, Universidade do Porto, Rua das Estrelas, 4150-762 Porto, Portugal \\ e-mail: papaderos@astro.up.pt
}

Received 14 May 2012 / Accepted 29 August 2012

\begin{abstract}
Context. Blue compact galaxies (BCG) are narrow emission-line systems that undergo a violent burst of star formation. They are compact, low-luminosity galaxies, with blue colors and low chemical abundances, which offer us a unique opportunity to investigate collective star formation and its effects on galaxy evolution in a relatively simple, dynamically unperturbed environment. Spatially resolved spectrophotometric studies of BCGs are essential for a better understanding of the role of starburst-driven feedback processes on the kinematical and chemical evolution of low-mass galaxies near and far.

Aims. We carry out an integral field spectroscopy (IFS) study of a sample of luminous BCGs, with the aim to probe the morphology, kinematics, dust extinction, and excitation mechanisms of their warm interstellar medium (ISM).

Methods. We obtained IFS data for five luminous BCGs with VIRUS-P, the prototype instrument for the Visible Integral Field Replicable Unit Spectrograph, attached to the $2.7 \mathrm{~m}$ Harlan J. Smith Telescope at the McDonald Observatory. VIRUS-P consists of a square array of 247 optical fibers, which covers a $109^{\prime \prime} \times 109^{\prime \prime}$ field of view, with a spatial sampling of 4". 2 and a 0.3 filling factor. We observed in the 3550-5850 $\AA$ spectral range, with a resolution of $5 \AA$ FWHM. From these data we built two-dimensional maps of the continuum and the most prominent emission-lines ([O II] $\lambda 3727, \mathrm{H} \gamma, \mathrm{H} \beta$ and [O III] $\lambda 5007$ ), and investigated the morphology of diagnostic emission-line ratios and the extinction patterns in the ISM as well as stellar and gas kinematics. Additionally, from integrated spectra we inferred total line fluxes and luminosity-weighted extinction coefficients and gas-phase metallicities.

Results. All galaxies exhibit an overall regular morphology in the stellar continuum, while their warm ISM morphology is more complex: in II Zw 33 and Mrk 314, the star-forming regions are aligned along a chain-structure; Haro 1, NGC 4670 and III Zw 102 display several salient features, such as extended gaseous filaments and bubbles. A significant intrinsic absorption by dust is present in all galaxies, the most extreme case being III $\mathrm{Zw}$ 102. Our data reveal a plethora of kinematical patterns, from overall regular gas and stellar rotation to complex velocity fields produced by structurally and kinematically distinct components.
\end{abstract}

Key words. galaxies: starburst - galaxies: dwarf - galaxies: abundances - galaxies: kinematics and dynamics

\section{Introduction}

Blue compact galaxies (BCGs) are low-luminosity $\left(M_{B}\right.$ ranges from -12 to -21$)$ and low-metallicity $(7.0 \leq 12+\log (\mathrm{O} / \mathrm{H}) \leq$ 8.4) systems that have optical spectra similar to those of H II regions in spiral galaxies (Thuan \& Martin 1981; Kunth \& Östlin 2000). With star formation rates between 0.1 and $1 M_{\odot} \mathrm{yr}^{-1}$ (Fanelli et al. 1988; Hunter \& Elmegreen 2004), they represent the most extreme manifestation of star-forming (SF) activity in late-type dwarf galaxies in the local Universe.

The BCGs are of paramount importance in extragalactic astronomy and observational cosmology research, because they hold key clues for understanding fundamental topics such as galaxy formation and evolution, and the ignition and selfregulation of SF activity in relatively isolated late-type galaxies.

They represent an unparalleled link to the early Universe, since in the commonly accepted framework of a cold dark matter (CDM) Universe, structure formed hierarchically, with smallscale objects (low-mass halos) collapsing first at relatively high redshifts. About two percent of the BCGs have very low metallicities, $(12+\log (\mathrm{O} / \mathrm{H}) \leq 7.65)$; these objects, often referred to as extremely metal deficient (XBCD) galaxies (Papaderos et al. 2008), constitute the best local analogs to the distant subgalactic units from which larger galaxies are formed (Papaderos et al. 1998; Kniazev et al. 2004). Furthermore, the more luminous BCGs (or different subsamples of them) have been regarded as the local counterparts of different high-redshift SF galaxy populations (Mas-Hesse et al. 2003; Grimes et al. 2009). Detailed analyses of these nearby objects are essential for interpreting the observations of the more distant SF galaxy population, because their proximity allows for studies focused on their stellar content, kinematics, and abundances with an accuracy and spatial resolution that cannot be achieved at higher redshifts (Cairós et al. 2009a,b, 2010).

Moreover, low-luminosity systems are the best laboratories to study the star-formation process. Without spiral density waves to act as a triggering mechanism for star formation, these galaxies enable us to investigate in an unbiased manner all of the 
other factors that trigger and control SF activities. In addition, BCGs offer a unique opportunity to test star formation models at very low metallicities, similar to those of the early Universe.

Despite of the strong interest gained for BCGs in the last decades, several key questions in the field, namely, the evolutionary status of BCGs, their star forming history (SFH) or the mechanisms that trigger their star-forming activity, are still open.

One of the reasons is probably that most of the BCG work published so far deal with analyses of samples by means of surface photometry (Papaderos et al. 1996; Cairós et al. 2001a,b; Doublier et al. 1997, 1999; Gil de Paz et al. 2003; Gil de Paz \& Madore 2005), and spectroscopic studies typically only cover the brightest SF regions of these systems. Studies combining surface photometry with spatially resolved spectroscopy and spectral synthesis that aim at a quantitative study of the formation history of stellar populations in BCGs are scarce.

That very few spectrophotometric analyses can be found in the literature, and virtually all of them focused on one single object (Guseva et al. 2003a,b,c; Cairós et al. 2002, 2007), is essentially due to the prohibitively long observing time that conventional observational techniques require: acquiring images in several broad-band and narrow-band filters, plus a sequence of long-slit spectra sweeping the region of interest, translate into observing times of a few nights per galaxy, making analyses of statistically meaningful samples not feasible in terms of observing time. Moreover, such observations usually suffer from varying instrumental and atmospheric conditions, which complicates combining all these data even more.

Integral field spectroscopy (IFS) offers a way to approach spectrophotometric BCG studies in a highly effective manner (Izotov et al. 2006a; García-Lorenzo et al. 2008; Vanzi et al. 2008; James et al. 2009; Cairós et al. 2009a,b, 2010). The IFS provides simultaneous spectra of each spatial resolution element under identical instrumental and atmospheric conditions, which is not only a more efficient way of observing, but also guarantees the homogeneity of the dataset. From only one integral field unit (IFU) frame we can produce a series of broadband images in many filters, narrow-band images in a large set of bands, as well as derive an extensive collection of observables from the spectrum (e.g. line indices). In terms of observing time, IFS observations of BCGs are one order of magnitude more efficient than traditional observing techniques, implying that now spectrophotometric studies of large samples of BCG galaxies have become viable.

The work presented here is part of the IFS-BCG survey, a challenging long-term project that aims to perform an exhaustive spectrophotometric survey of a large sample of BCGs by means of IFS. The main scientific goals of this projects include i) to distinguish and characterize the different stellar populations in BCGs (e.g. constrain their ages, initial mass function and metallicity); ii) to investigate the evolutionary status of the galaxies and constrain their SFH; iii) to probe the mechanism responsible for the actual burst of star formation; iv) to analyze the feedback effects between massive stars and the interstellar medium (ISM) in dwarf galaxies; v) to investigate the recent suggestion that some BCGs harbor active galactic nuclei (AGN) associated with intermediate-mass black holes (Izotov et al. 2010); vi) to provide an accurate dataset of photometric and spectroscopic parameters for a large sample of nearby SF dwarf galaxies, the essential template for understanding the results of the investigations at intermediate and high- $z$.

This is the fourth in a series of papers presenting the findings of the project, in which we report on results derived for five luminous BCGs observed with VIRUS-P. In the first and second papers of the series (Cairós et al. 2009a,b) we illustrated the full potential of this study by showing results on two representative BCGs, Mrk 1418 and Mrk 409, both observed with the Potsdam multi-aperture spectrophotometer (PMAS), attached at the 3.5-m telescope at Calar Alto Observatory. A more detailed description of the project objectives, IFU observation and reduction techniques and results for another eight objects observed with PMAS, was presented in Cairós et al. (2010).

This paper is structured as follows: in Sect. 2 we describe the observations, the data reduction process and the method employed to derive the maps. In Sect. 3 we present the main outcomes of the work, that is, the flux, line ratio, and velocity maps, as well as the results derived from the analysis of the integrated spectra. Finally, the main findings for each galaxy are discussed in Sect. 4 and are summarized in Sect. 5.

\section{Observations and data processing}

\subsection{Galaxy sample and observations}

The whole survey includes the mapping of about 40 galaxies, chosen so that they cover the wide range of luminosities $(-12 \geq$ $\left.M_{B} \geq-21\right)$ and metallicities $(7.0 \leq 12+\log (\mathrm{O} / \mathrm{H}) \leq 8.4)$ found among BCGs. We also took care that the different morphological classes observed among BCGs were adequately represented in the sample (Cairós et al. 2001a).

In this paper we present results for five luminous BCGs (Bergvall \& Östlin 2002; Cairós et al. 2002). These objects are the most luminous $\left(M_{B} \leq-17\right)$, and typically also the more irregular BCGs; they tend to have a substantial contribution of older stars, significant amounts of dust and very distorted starburst morphologies (Cairós et al. 2003; Bergvall 2012). As we have already stated in the introduction, these luminous BCGs have a major relevance in cosmological studies because they (or different subsamples of them) have been regarded as the local counterparts of different galaxy populations at higher redshifts $(z \approx 2-6)$, such as Lyman-break galaxies (Grimes et al. 2009) or Lyman- $\alpha$ emitters (Mas-Hesse et al. 2003).

The basic data for the galaxies and the complete log of the observations are listed in Tables 1 and 2, respectively. $B$-band images of the galaxies are displayed in Fig. 1. The IFS of III Zw 102 and Mrk 314 have been previously published by García-Lorenzo et al. (2008), who studied the central 33". $6 \times$ $29 . ' 4$ of III Zw 102 and $16^{\prime \prime} \times 12^{\prime \prime}$ of Mrk 314 using INTEGRAL at the William Herschel Telescope. In both cases, the much smaller field of view (FOV) of INTEGRAL allows only for a partial mapping of the starburst regions.

Spectral data for the five galaxies listed in Table 1 were collected using the VIRUS-P IFU spectrograph (Hill et al. 2008), working at the $2.7 \mathrm{~m}$ Harlan J. Smith Telescope at the McDonald Observatory (TX, USA). VIRUS-P is the prototype instrument for VIRUS (=Visible Integral-field Replicable Unit Spectrograph), a massively replicated IFU consisting of 150 units like VIRUS-P, which will be installed on the $9.2 \mathrm{~m}$ Hobby-Eberly Telescope (Hill et al. 2006, 2007).

VIRUS-P (with the VP-1 IFU bundle used in these observations) consists of a square array of 247 optical fibers (of which three were dead in the configuration used), which samples a $109^{\prime \prime} \times 109^{\prime \prime}$ FOV with a 0.3 filling factor. Each fiber is circular and has a diameter of 4 ". 2 on the sky. We observed in the spectral range $3550-5850 \AA$, with a spectral resolution (FWHM) of $\approx 5 \AA\left(340 \mathrm{~km} \mathrm{~s}^{-1}\right.$ at $\left.\mathrm{H} \beta\right)$.

Observations were carried out during two observing runs, in 2007 October and in 2008 March. During the latter run, 
Table 1. Galaxy sample.

\begin{tabular}{|c|c|c|c|c|c|c|c|c|}
\hline $\begin{array}{l}\text { Galaxy } \\
\text { (1) }\end{array}$ & Other designations & $\begin{array}{c}\text { RA } \\
(2000) \\
(3)\end{array}$ & $\begin{array}{c}\text { Dec } \\
(2000) \\
(4)\end{array}$ & $\begin{array}{l}m_{B} \\
(5) \\
\end{array}$ & $\begin{array}{c}D \\
(\mathrm{Mpc}) \\
(6)\end{array}$ & $\begin{array}{c}M_{B} \\
\text { (mag) } \\
\text { (7) }\end{array}$ & $\begin{array}{c}\text { Morph } \\
\text { (8) }\end{array}$ & $\begin{array}{c}A_{\mathrm{V}} \\
(\mathrm{mag}) \\
(9)\end{array}$ \\
\hline II Zw 33 & UGCA 102, Mrk 1094 & 051048.1 & -024054 & $14.57^{a}$ & 37.4 & -18.29 & $\mathrm{Ch}$ & 0.288 \\
\hline Haro 1 & NGC 2415, UGC 3930 & 073656.7 & 351431 & $12.61^{a}$ & 52.9 & -21.00 & Ext & 0.117 \\
\hline NGC 4670 & UGC 7930, Arp 163, Haro 9 & 124517.2 & 270732 & $13.02^{b}$ & 22.4 & -18.73 & Nuc & 0.041 \\
\hline Mrk 314 & NGC 7468, UGC 12329 & 230259.2 & 163619 & $13.78^{a}$ & 29.0 & -18.53 & $\mathrm{Ch}$ & 0.234 \\
\hline III Zw 102 & NGC 7625, UGC 12529, Arp 212 & 232030.1 & 171332 & $12.50^{a}$ & 23.1 & -19.31 & Ext & 0.069 \\
\hline
\end{tabular}

Notes. Columns (3) and (4): units of right ascension are hours, minutes, and seconds, and units of declination are degrees, arcminutes, and arcseconds. Column (5): $B$-band magnitudes: ${ }^{(a)}$ asymptotic photometry obtained by extrapolating the growth curves, and corrected for galactic extinction (Cairós et al. 2001a). The asymptotic magnitudes listed in Cairós et al. (2001a) were corrected for Galactic extinction following Burstein \& Heiles (1982); here they were recomputed using the Schlafly \& Finkbeiner (2011) extinction values; ${ }^{(b)}$ integrated magnitudes from Gil de Paz et al. (2003), corrected for Galactic extinction. Column (6): distances, computed assuming a Hubble flow, with a Hubble constant $H_{0}=73 \mathrm{~km} \mathrm{~s}^{-1} \mathrm{Mpc}^{-1}$, and taking into account the influence of the Virgo cluster, the Great Attractor and the Shapley supercluster, are taken from NED (http://nedwww.ipac. caltech.edu). Column (7): absolute magnitude in the $B$ band, computed from the tabulated $B$-band magnitude and distance. Column (8): morphological classification following Cairós et al. (2001a). Column (9): galactic extinction, from the Schlafly \& Finkbeiner (2011) recalibration, as listed in NED.

Table 2. Log of the observations.

\begin{tabular}{lccccc}
\hline \hline Galaxy & Date & $\begin{array}{c}\text { Exp. time } \\
(\mathrm{s})\end{array}$ & Airmass range & $\begin{array}{c}\text { Seeing } \\
(\operatorname{arcsec})\end{array}$ & $\begin{array}{c}\text { Spatial scale } \\
\left(\mathrm{pc} \operatorname{arcsec}^{-1}\right)\end{array}$ \\
\hline II Zw 33 & Oct. 2007 & 3600 & $1.20-1.40$ & $2.2-2.4$ & 760 \\
Haro 1 & Mar. 2008 & 3600 & $1.01-1.10$ & $2.5-3.2$ & 1080 \\
NGC 4670 & Mar. 2008 & 4800 & $1.00-1.13$ & $2.0-3.0$ & 460 \\
Mrk 314 & Oct. 2007 & 5400 & $1.03-1.20$ & $1.8-2.0$ & 590 \\
III Zw 102 & Oct. 2007 & 4200 & $1.03-1.25$ & $2.0-2.8$ & 470 \\
\hline
\end{tabular}
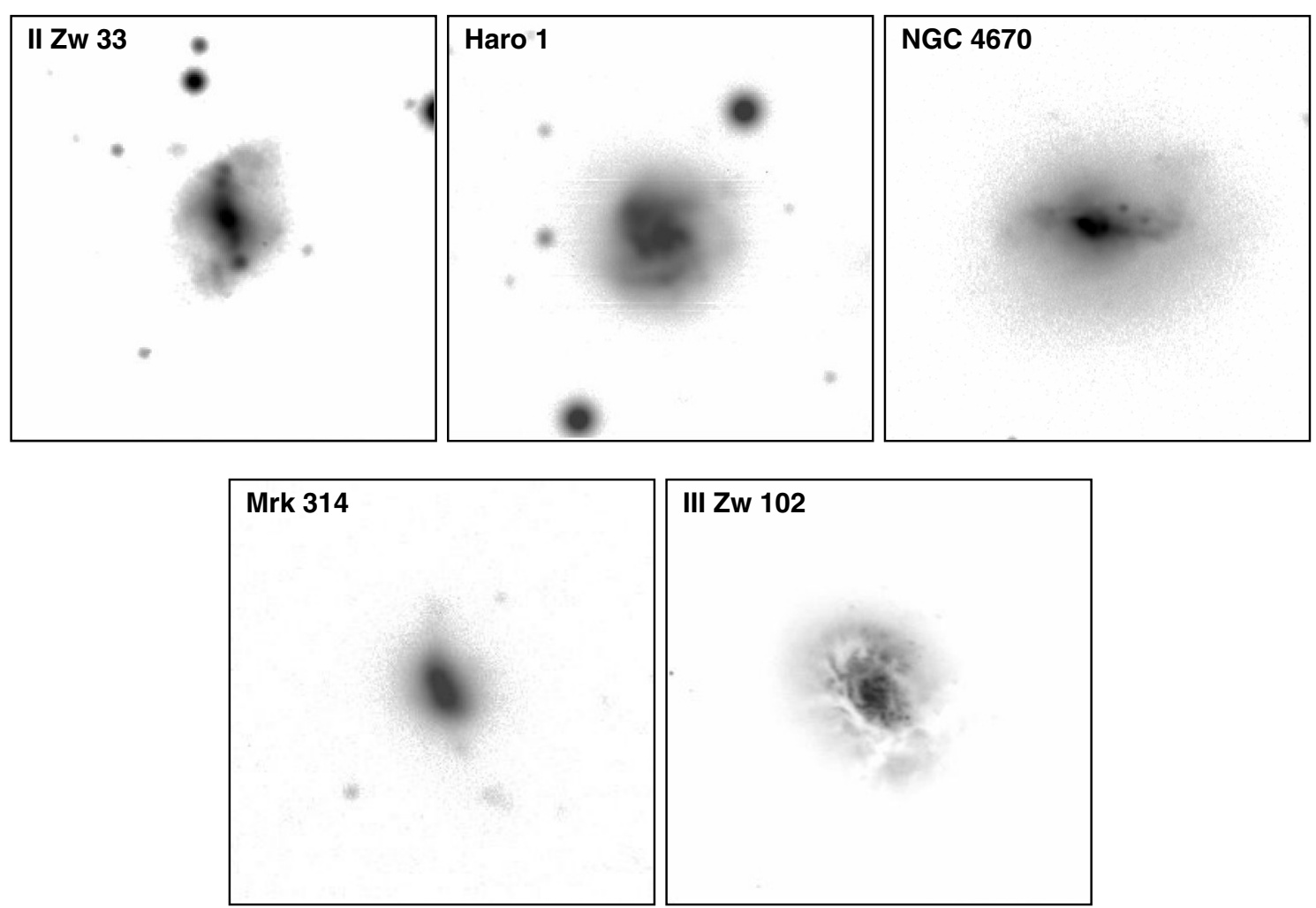

Fig. 1. $B$-band images of the galaxies (in parenthesis the instrument/telescope at which the images were obtained): II Zw 33 (CAFOS at the $2.2 \mathrm{~m}$ CAHA, Cairós et al. 2001b); Haro 1 (Prime Focus at 3.5-m CAHA, Cairós et al. 2001b); NGC 4670 (60 inch telescope on Mt. Palomar, Gil de Paz et al. 2003); Mrk 314 (prime Focus at the 3.5-m CAHA, Cairós et al. 2001b) and III Zw 102 (ALFOSC/NOT, unpublished). North is up, east to the left. The FOV is $109^{\prime \prime} \times 109^{\prime \prime}$ (the FOV of the VIRUS-P instrument). All images are shown in logarithmic scale. 
the spectra of five fibers fell off the chip due to camera alignment problems, and therefore only 239 fibers were usable.

We followed the same strategy in the two observing campaigns. Calibration frames consisted of bias frames, sky-flats and comparison lamps taken at the beginning and at the end of the night. Sky-flats are required to define and trace the fibers (apertures) on the detector as well as to perform the throughput correction. Spectra of emission-line lamps (Hg-Cd lamp in our case) are needed to carry out the wavelength calibration. Because VIRUS-P is an instrument with practically no flexures, comparison lamp spectra taken once during the night will suffice, and no arc exposures interspersed with the science observations are required.

As the filling factor of the IFU is $30 \%$, three dithered exposures were taken to ensure a coverage of about $90 \%$ of the FOV. The standard observing sequence consists of a set of three exposures (of 20 to $30 \mathrm{~min}$ each) at each of the three positions (the telescope offsets are first $33^{\prime \prime} 9$ west, 2 '. 1 south and then 3 '. 75 east, 2'. 1 south).

Flux spectrophotometric standards were also observed, albeit using a six-position dither pattern, which guarantees the collection of (almost) their total flux. The spectrophotometric standard stars $\mathrm{BD}+25 \mathrm{~d} 4655, \mathrm{G} 191-\mathrm{B} 2 \mathrm{~B}$ and $\mathrm{BD}+40 \mathrm{~d} 4032$ were observed during the October run and Feige 34 and $\mathrm{BD}+33 \mathrm{~d} 2642$ in the March run.

\subsection{Data reduction}

The data were processed using standard $\mathrm{IRAF}^{1}$ tasks.

The first step in the data reduction was to remove the bias pedestal level, whose value was computed using the overscan regions in the science frames and was subtracted. Because the bias frames showed some smooth large-scale structure, we averaged all of them to obtain a master bias, from which we subtracted its mean value. This master bias was then subtracted from all the overscan-corrected frames.

Next, apertures were defined and traced on the detector. The apertures need to be traced and defined in well-exposed frames: sky-flats taken at the beginning or at the end of the night were used for that. We defined the apertures using the IRAF task apall; this task first finds the centers of each fiber (the emission peak) along the spatial axis at some specified position, and then the user is prompted for the size of the extraction window, which we set to seven pixels, a width that in our dataset maximized the overall signal-to-noise ratio (cross-talk is negligible, see Murphy et al. 2011). The apertures were then traced by fitting a polynomial to the centroid along the dispersion axis. A fifth-degree Legendre polynomial was found to provide good fits, with a typical rms of about 0.02 pixels.

Once the apertures were defined and traced in the continuum frames, we again used apall to extract them in all science frames. The extraction consists of summing the pixels along the spatial direction into a one-dimensional spectrum for each fiber. The output is thus a "row-stacked spectrum" (RSS) of the 244 spaxels (239 for the 2008 March run).

We then performed the wavelength calibration. First, we identified several emission features of known wavelength in a reference fiber in the comparison spectra. Second, using identify, a polynomial was fitted along the dispersion direction; the

\footnotetext{
1 IRAF is distributed by the National Optical Astronomy Observatories, which are operated by the Association of Universities for Research in Astronomy, Inc., under cooperative agreement with the National Science Foundation.
}

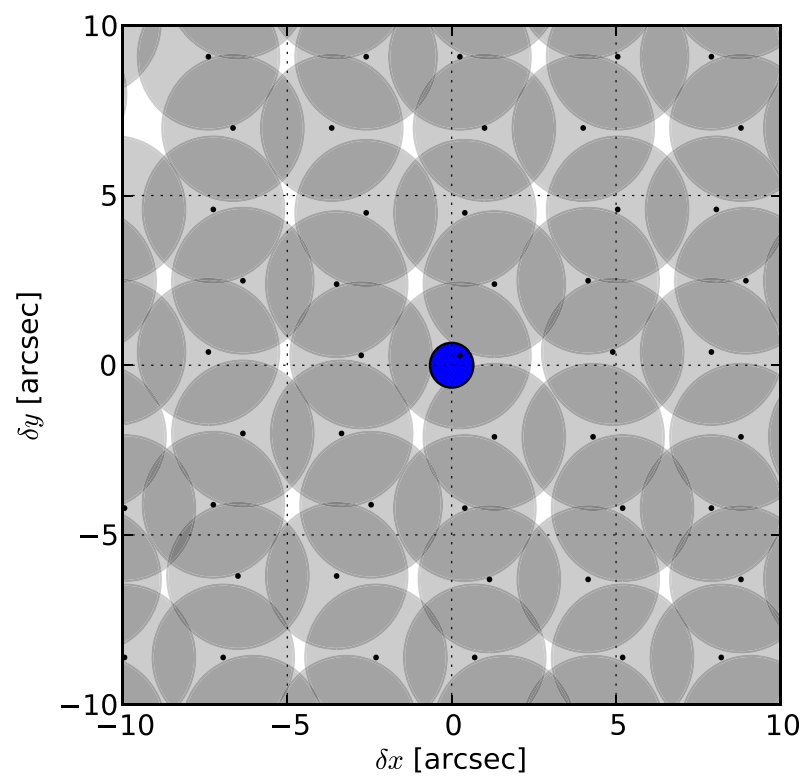

Fig. 2. Size and position of the fibers, centered on the position of the star (G 191-B2B). The fiber diameter is $4{ }^{\prime \prime}$. 2 , the distance between adjacent fibers in the same exposure is 7".5. The blue circle shows the computed $F W H M$ of this star $\left(\simeq 1^{\prime \prime} .4\right)$.

standard deviation (rms) of the polynomial fit gives an estimate of the uncertainty in the wavelength calibration. We obtained a typical rms of about $0.01 \AA$ by fitting a fifth-degree Legendre polynomial. A total of 13 lines were fitted. Finally, with reidentify we identified the emission lines in all remaining fibers of the arc frame, using the selected one as a reference.

Subsequently we corrected for throughput (spaxel-to-spaxel variations) by using the sky flat exposures. This step was carried out with the task msrespl.

In each spectrum, the sky background was computed by taking the median of $\sim 40-50$ spaxels in the periphery of the FOV where the galaxy line and continuum emission is negligible and was subtracted. The spectrum was then corrected for atmospheric transparency variations, gauged by means of the flux of the guide star in the guider-saved frames, as explained in Blanc et al. (2009), and for atmospheric extinction (the guide star exposures were also used to estimate the seeing during the observations, see Table 2).

The three spectra for each dithering position were averaged together using the IRAF task imcombine, and then stacked to form a final spectrum of 732 rows (717 for the 2008 March observations).

\subsection{Flux calibration}

Spectra of several spectrophotometric standard stars were obtained and reduced in the same way as the galaxy spectra.

The only remarkable difference is that the standard stars were observed with a six-position dithering pattern, which ensures that only about $3 \%$ of FOV is "lost" in inter-fibers gaps, while about two thirds of the area are covered by two overlapping fibers, as shown in Fig. 2. Combined with the PSF undersampling, this dithering pattern does not allow an accurate direct flux estimation by simple summation of all relevant spaxels.

If we summed all spaxels with sufficiently strong stellar continuum (typically about 30 spaxels in the row-stacked final spectrum), we would overestimate the actual stellar spectrum, because of the dithering geometry. Moreover, the overestimation factor is in principle wavelength-dependent, because of both the 


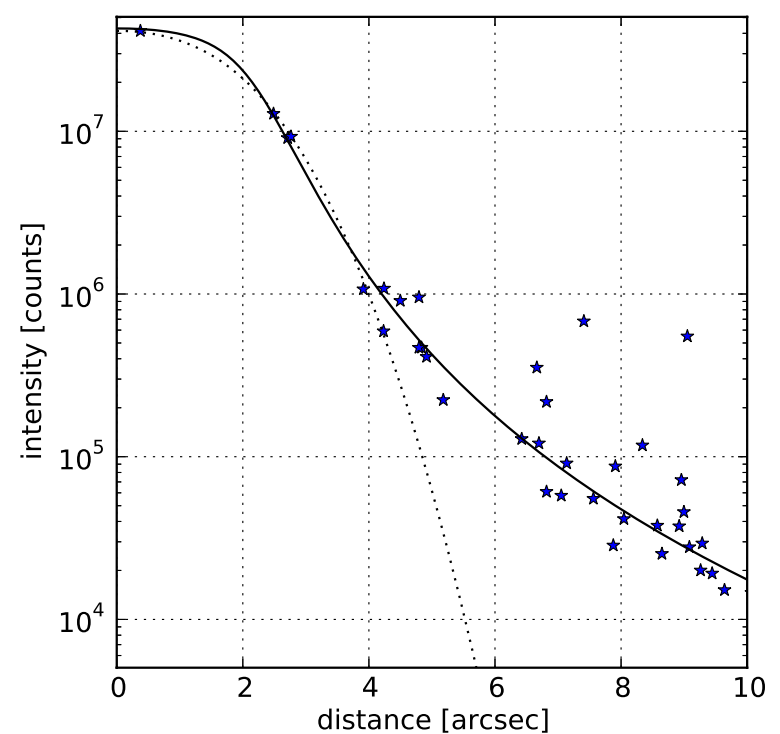

Fig. 3. Measured and fitted intensity for the source G 191-B2B. The $X$-axis shows the distance to the fitted source center, $Y$ the intensity. Stars indicate the positions of the fiber centers. The curves are the best Moffat (solid) and Gaussian (dotted) fits (both integrated over the fiber area).

increase of the stellar PSF width toward bluer wavelengths and the shift of the light centroid due to differential atmospheric refraction.

To better understand this, we can think of a hypothetical star with an extremely narrow PSF whose blue centroid happens to fall on one of the inter-fibers gaps while the red centroid falls on a spatial location covered by two overlapping fibers: we would measure very little or zero blue flux, while the red flux would be overestimated by a factor of two.

To assess the strength of this effect, we simulated the response of each fiber and performed a $\chi^{2}$ fit to determine the PSF parameters. The fiber response was calculated by a Monte Carlo integration of the PSF over the circle area of the fiber. For the PSF, a Moffat profile was chosen to follow the instrument response to the outer wings, as shown in Fig. 3. Fit parameters were the total flux, source position, and the PSF width $\alpha$ and exponent $\beta$. The Moffat fit was performed individually for each wavelength bin.

We found a tight correlation between the Moffat function parameters and wavelength, as depicted in Fig. 4; also, the observed shift of the PSF centroid agrees well with the shift computed theoretically from the airmass and the parallactic angle of the spectrophotometric standard stars spectra.

However, the overestimation factor turns out to have only a very weak $(<0.03 \mathrm{mag}$ ) dependence on wavelength (Fig. 5), because of the relatively poor seeing, which spreads out the star flux over many spaxels, and because the standard stars were observed at low airmasses.

To derive the final flux calibration function, we summed all spaxels with sufficiently strong signal in the stacked spectrum for each spectrophotometric standard star and applied the overestimating factor correction. Then the IRAF tasks standard and sensfunc were used to compute the calibration function, after combining the data for the different spectrophotometric stars.

By comparing the sensitivity curves for the different standard stars in the two observing runs, we can estimate that the relative uncertainty on the calibration factor is generally less than $2 \%$, except at the blue end of the spectrum, where the uncertainty increases to about $7 \%$.

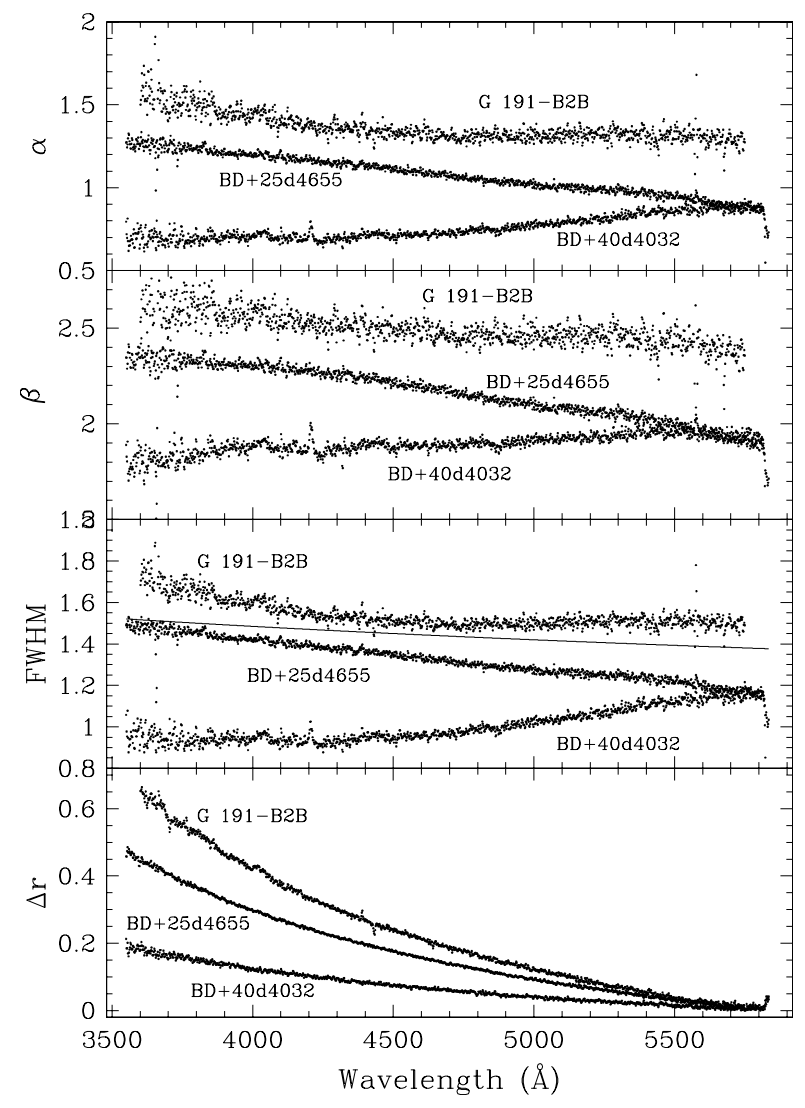

Fig. 4. Moffat $\alpha$ and $\beta$ parameters are plotted as a function of the wavelength for three spectrophotometric standards. The bottom panels show the computed FWHM (the solid line represents the $F W H M \propto \lambda^{-0.2}$ relation) and the shift of the centroid with respect to a reference point at $\lambda=5750 \AA$.

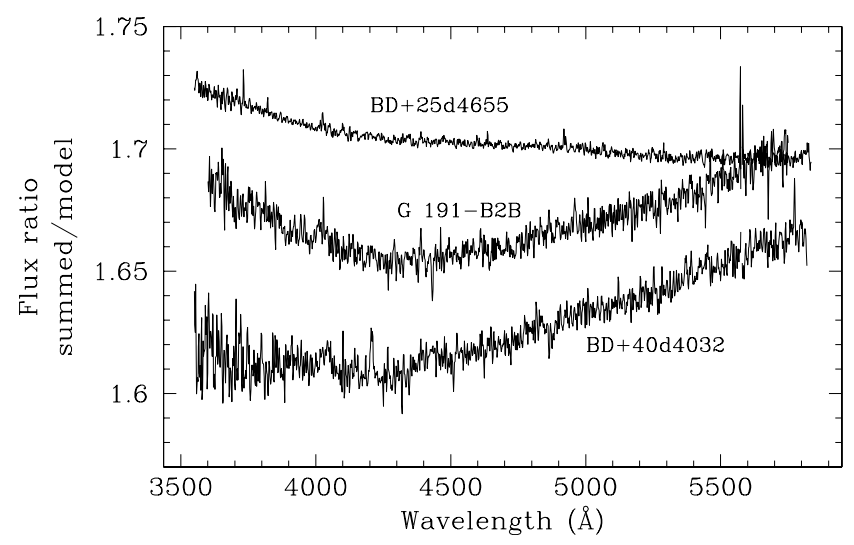

Fig. 5. Ratio between the flux computed by a direct sum of the spectra of all spaxels with sufficiently good signal and the flux derived by the Moffat fit to the data as a function of wavelength for three spectrophotometric standard stars. The direct integration overestimates the stellar flux by a factor of about $5 / 3$, which depends very little on wavelength.

Because the diameter of the fibers and the average seeing values are much higher than differential atmospheric refraction (DAR) effects, no correction for DAR was applied to the data.

\subsection{Emission-line fit}

\subsubsection{Recovering the pure emission-line spectrum}

Measuring the emission-line properties (e.g. fluxes, equivalent widths, or velocity dispersion) provides much information about 
the nebula physical conditions and the properties of the ionizing stellar population. However, and in particular when dealing with $\mathrm{SF}$ galaxies, deriving accurate emission-line fluxes and equivalent widths is not free of problems. At any given spatial position, the observed galaxy spectrum is the sum of the nebular and stellar emission; underlying stellar absorption can under certain circumstances dramatically decrease the measured intensity and equivalent width of Balmer emission lines.

Therefore, to obtain precise measurements of the emissionline parameters of the ionized gas, the flux emitted by the stellar population must be modeled and subtracted. For the IFU data, this process must be performed for every spaxel, i.e., the technique chosen to model the stellar population has to be applied to each individual spectrum of the IFS mosaic.

To model the stellar population, we used the penalized pixelfitting (pPXF) method ${ }^{2}$ developed by Cappellari \& Emsellem (2004); the pPXF software allows one to simultaneously fit the optimal linear combination of stellar templates to the observed spectrum and to derive the stellar kinematics, using a maximum likelihood approach to suppress noise solutions. We used the MILES stellar library ${ }^{3}$ (Sánchez-Blázquez et al. 2006), which contains a total of 985 stars spanning a wide range in stellar age and metallicity.

As a first step, we produced a high signal-to-noise integrated spectrum for every galaxy by adding all spectra of the science spaxels. With this spectrum we found the optimal stellar template for every object, i.e., the selected stars from the MILES library that produce the best fit. The fit to the stellar continuum was made after masking the gas emission-lines expected in the spectral range under study, as well as residuals from skyline subtraction. The number of stars combined to create the optimal stellar template for each galaxy was 17 stars for II Zw 33, 20 stars for Haro 1, 22 stars for NGC 4670, 20 stars for Mrk 314, and 25 stars for III Zw102.

After that, the pPXF method was run on the spectra of each individual spaxel, using the optimal stellar template and allowing the weights of each star to vary. The application of the optimal stellar template to each individual spectrum speeds the fitting process up considerably. Because the automatization of this process might lead to bad fittings, a careful individual inspection by eye was made.

Once the stellar continuum was successfully reproduced by a sum of templates for every spaxel, it was subtracted from the original spectrum to obtain the pure emission-line spectrum.

We remark here that the fit to the stellar continuum is not meant to provide a physical description of the stellar populations of which the galaxy consists, a topic which is outside the scope of this paper, but that the purpose of this modeling is to obtain a good representation of the underlying stellar continuum to decouple it from the emission lines produced by the ionized gas, and to derive more accurate values of emission-line fluxes.

The stellar continuum fit is possible only in the central regions of the galaxies, while it fails, producing meaningless results, in the outer regions where the signal-to-noise ratio of the continuum is too low.

\subsubsection{Fitting the lines}

Emission-line fluxes for every spaxel were measured on the pure emission-line spectrum. However, the line emission extends well

\footnotetext{
2 URL: http://wWw-astro.physics.ox.ac.uk/ mxc/idl/

3 URL: http://miles.iac.es/
}

beyond the galaxy region in which the pure emission-line spectrum could be computed.

To compute the fluxes in the spaxels outside these regions, the original stacked spectra were used by fitting the continuum, typically $30-50 \AA$ on both sides, by a straight line.

To measure the relevant parameters of the emission lines (position, flux, and width), they were fitted by single Gaussians. The fit was produced with the Levenberg-Marquardt nonlinear fitting algorithm implemented by Markwardt in the mpfitexpr IDL library ${ }^{4}$. The [O III] $\lambda \lambda 4959,5007$ doublet was fitted imposing that both lines have the same redshift and width. Uncertainties on the fit output parameters were computed by adopting a simplified Poissonian model on the non-fluxcalibrated spectra, where the readout noise was set equal to the rms of the nearby continuum, and the gain set to 1 .

Criteria such as flux, error on flux, velocity, and width were used for a first automatic assessment of whether to accept or reject a fit. For instance, lines with too narrow (narrower than the instrumental width) or too wide widths were flagged as rejected, as were lines with an error on the flux higher than about $10 \%$ of the flux (the exact limits depended on the specific line and on the overall quality of the spectrum). These criteria were complemented by a visual inspection of all fits, which led to override the automated criteria decision in a few cases (by accepting a fit flagged as rejected, or vice versa).

\subsection{Creating the $2 D$ maps}

For each line and for each line parameter (for instance flux), the emission-line fit procedure yields a table with the fiber ID number, its coordinates, the measured value, and the acceptance/ rejection flag.

The Gaussian fit parameters for the continuum-subtracted and the original spectra were merged. For the $\mathrm{H} \gamma$ and the $\mathrm{H} \delta$ Balmer lines only the results from the continuum-subtracted spectra were used due to their strong absorption components.

Line ratios were computed by simply dividing the fluxes of the corresponding lines.

To display all these maps, we explored several options. One is to interpolate the data on a regularly spaced grid by means of an algorithm such as the Renka \& Cline (1984) two-dimensional interpolation method. However, our own experience is that the interpolation can produce spurious oscillations, especially over noncontiguous spaxels, and gives a false impression of a spatial resolution higher than the actual value.

An alternative is to represent each spaxel with measured data with a circle with the fiber's diameter at its spatial position. However, the irregular inter-fiber gaps (because the actual position of the fibers is slightly and randomly offset from the nominal one) distract the eye of the viewer and interfere with a comfortable and effective visual assessment of the global characteristics of the map.

The solution we adopted was to depict each fiber by means of an hexagon centered on the nominal (not the actual) position of each fiber (the mean difference between nominal and actual positions is about 0.4 with a maximum of 1 1.' 8 ). This we found to be the best compromise between a truthful representation of the data (because no rebinning or interpolation was performed: the only manipulation was to slightly change the fibers positions and shape) and a map that is both pleasing to the eye and easy to appraise and interpret.

4 URL: http://purl.com/net/mpfit 
II Zw 33
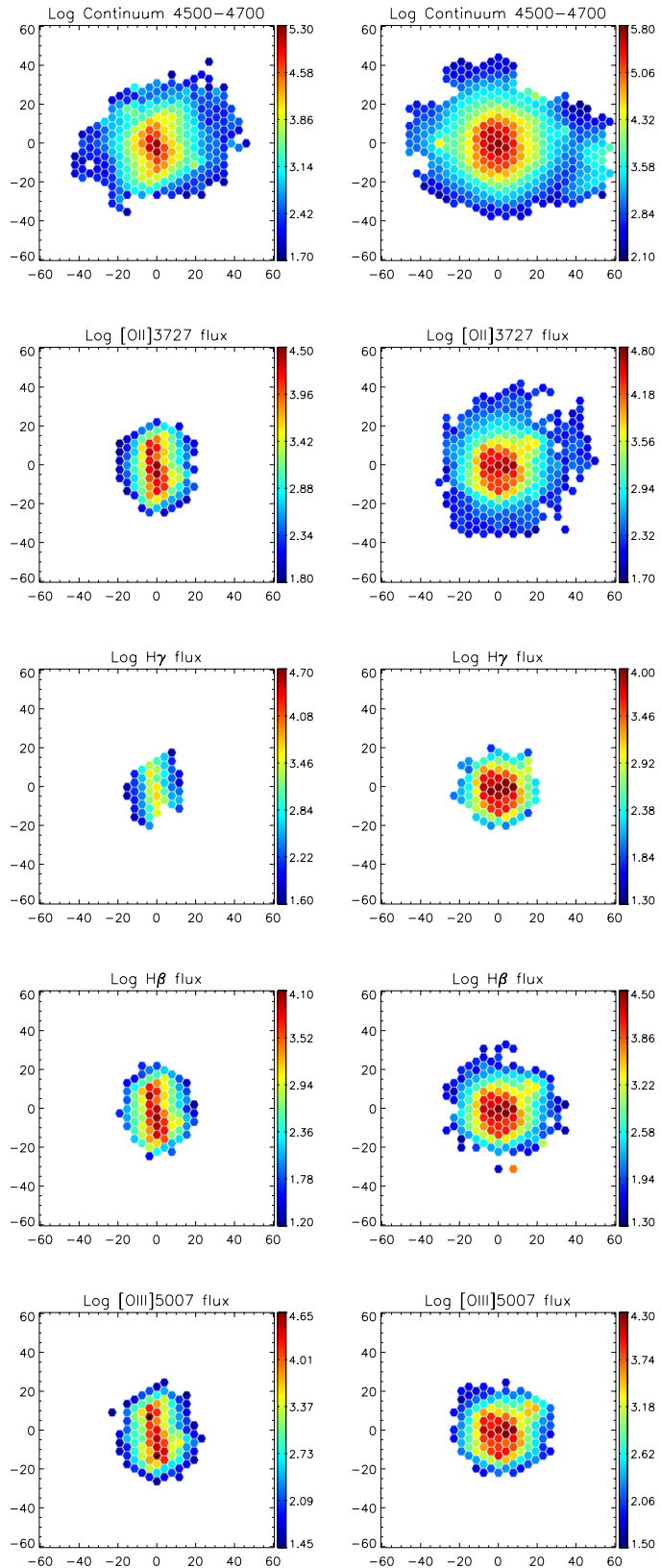

Fig. 6. Observed intensity maps (not reddening-corrected). First row: continuum emission between 4500 and $4700 \AA$ (a spectral region free from gas emission). Rows 2 to 5: emission-line flux maps derived from Gaussian fits to each line and spaxel (see text for details). Axis units are arcseconds; north is up, east to the left. All images are shown in logarithmic scale. Flux units are $10^{-18} \mathrm{erg} \mathrm{cm}^{-2} \mathrm{~s}^{-1}$. Spaxels where the blue Wolf-Rayet bump is visible are marked by stars in the [O III] map.

\section{Results}

\subsection{Emission-line and continuum intensity maps}

The IFS provides a simultaneous mapping of the light emitted by a galaxy in a wide spectral range. Accordingly, from one single exposure we can retrieve maps at a given wavelength or maps equivalent to narrow- or broad-band images within the whole observed range.

Following the steps described in Sect. 2.5, we built emissionline flux maps for the brightest observed emission-lines, namely [O II] $\lambda 3727, \mathrm{H} \gamma, \mathrm{H} \beta$, and [O III] $\lambda 5007$; these maps are displayed in Fig. 6 (rows 2 to 5).
Mrk 314

III Zw 102
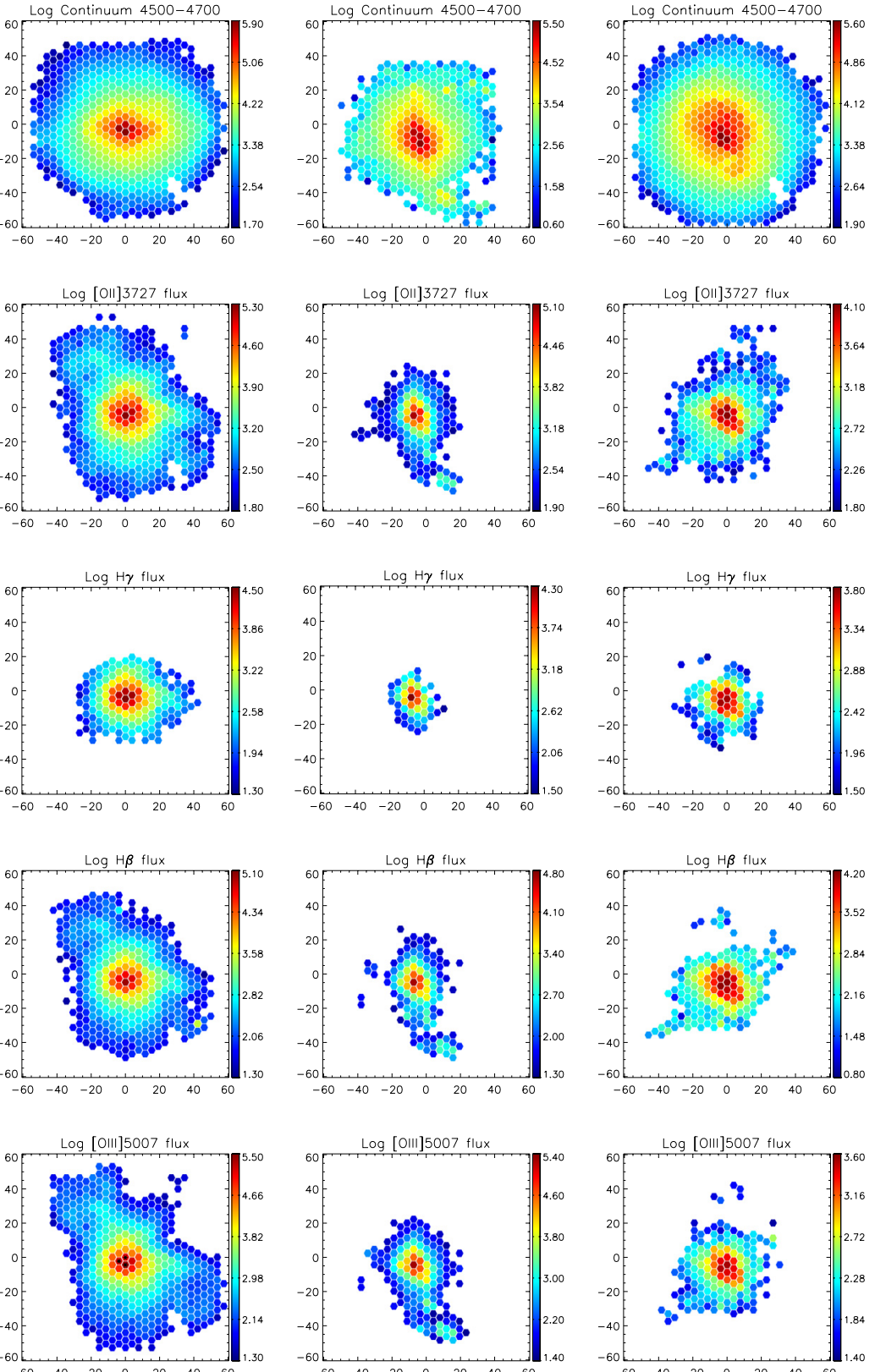
II Zw 33
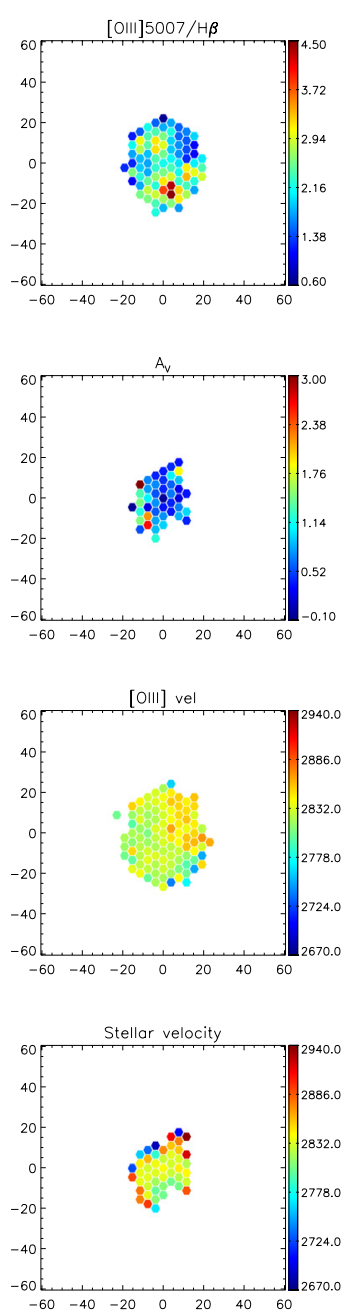

Haro 1
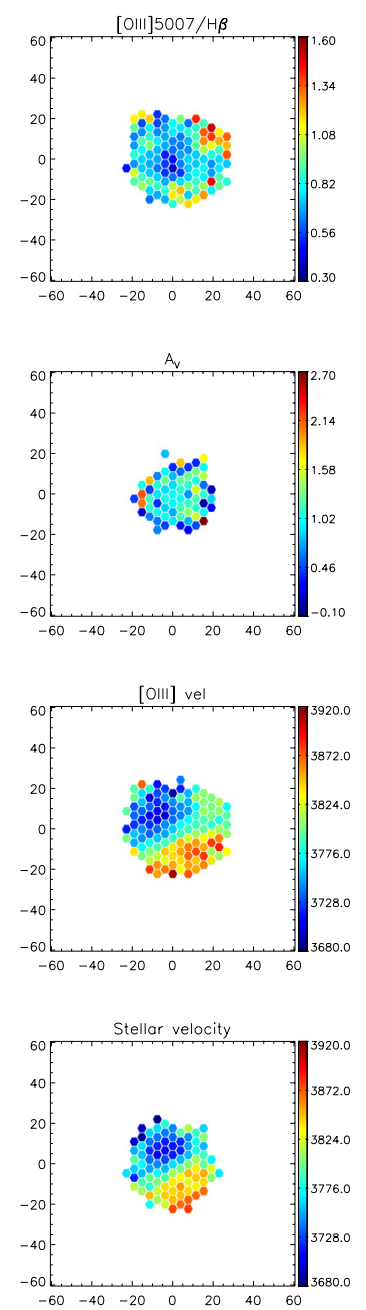

NGC 4670
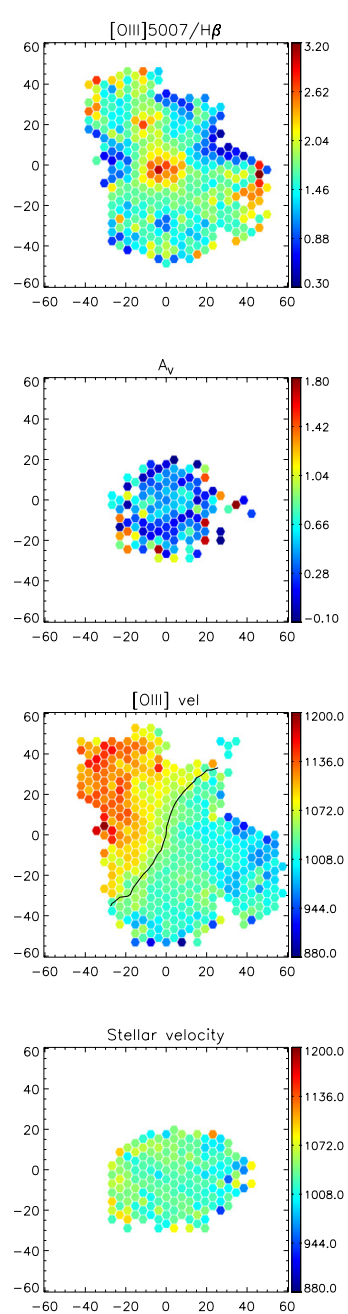

Mrk 314
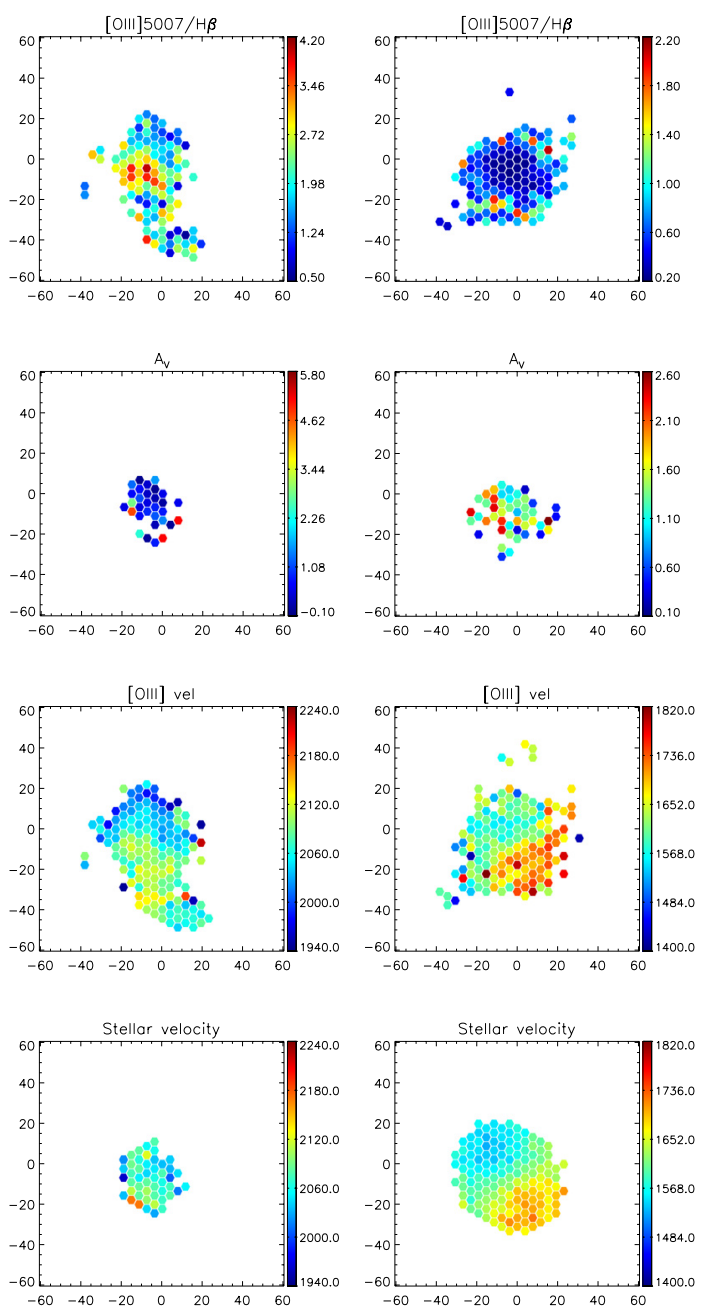

Fig. 7. First row: observed (not reddening-corrected) ionization ratio [O III] $\lambda 5007 / \mathrm{H} \beta$ for the five galaxies. Second row: interstellar extinction $A_{\mathrm{V}}$ map, computed from the $\mathrm{H} \beta / \mathrm{H} \gamma$ ratio. Row three: velocity field (in $\mathrm{km} \mathrm{s}^{-1}$ ) of the ionized gas [O III] $\lambda 5007$ emission line, except for III Zw 102 for which the $\mathrm{H} \beta$ velocity field is shown. Row four: stellar velocity field. Axis units are arcseconds. The solid line in the gas velocity map of NGC 4670 is the isovelocity contour at $V=1050 \mathrm{~km} \mathrm{~s}^{-1}$ (see text for details).

morphology in emission-lines; in II Zw 33 and Mrk 314, the $\mathrm{SF}$ regions are aligned to form a chain, while in Haro 1, NGC 4670 and III Zw 102 the ionized gas departs from the inner regions, giving rise to a complex pattern, where the presence of several bubbles and filaments is remarkable.

On the other hand, the continuum morphology is regular in the five objects (the jagged appearance of the Haro 1 continuum map is due to the presence of a few foreground stars, which have been masked, and a foreground galaxy at the west). In all galaxies, the continuum peak is located roughly at the center of the outer isophotes, and its position does not depend on the specific wavelength range used to build the continuum maps.

\subsection{Line ratio maps}

The first row of Fig. 7 displays the [O III] $\lambda 5007 / \mathrm{H} \beta$ maps for the five galaxies. This ratio essentially probes the hardness of the stellar radiation field within the nebula, and consequently high values of [O III] $\lambda 5007 / \mathrm{H} \beta$ are predicted when the ionization is produced predominantly by UV photons from OB stars (McCall et al. 1985). However, it is also sensitive to other factors such as the metallicity (Searle 1971), the distance from the ionizing source to the [O III] emitting layer (Stasińska 2009), or the presence of shocks (Dopita \& Sutherland 1995). Therefore the information provided by [O III] $\lambda 5007 / \mathrm{H} \beta$ is limited unless additional data regarding these other parameters are added.

For II Zw 33 and Mrk 314, the excitation maps roughly mimic the emission-line maps, the highest [O III] $\lambda 5007 / \mathrm{H} \beta$ values $(\geq 3)$, spatially coinciding with the highest peak in emissionlines, as usual in systems photoionized by hot stars.

Both Haro 1 and III $\mathrm{ZW} 102$ show more complex [O III] $\lambda 5007 / \mathrm{H} \beta$ patterns, with the highest values of the excitation localized in the galaxy periphery. This behavior, which is frequently found in spiral galaxies, has been interpreted as an abundance gradient (Smith 1975). Interestingly, Haro 1 and III Zw 102 are the two most luminous objects in the sample and lie both relatively far from the dwarf galaxy regime.

NGC 4670 shows a somewhat complex excitation morphology, with several [O III] $\lambda 5007 / \mathrm{H} \beta$ peaks, located in the central giant $\mathrm{H}$ II region and at the outer edge of the two larger filaments. For all five galaxies, the excitation levels are consistent with $\mathrm{H}$ II-like ionization. 
Interstellar extinction can be probed by comparing the observed ratios of hydrogen recombination lines with their theoretical values (Osterbrock \& Ferland 2006). In the optical domain, extinction maps are usually derived from the ratio $\mathrm{H} \alpha / \mathrm{H} \beta$ because it involves the strongest lines and is therefore the easiest to measure. In our case, lacking $\mathrm{H} \alpha$, we derived the extinction from the $\mathrm{H} \beta / \mathrm{H} \gamma$ line ratio; the extent of the extinction maps is dictated by the extent of the $\mathrm{H} \gamma$ maps (as said in Sect. 2.5 we only measured $\mathrm{H} \gamma$ in the continuum-subtracted spectra).

The extinction maps are shown in the second row of Fig. 7 using the $A_{\mathrm{V}}$ parameter. We adopted the Case B, lowdensity limit, $T=10000 \mathrm{~K}$ approximation, where the theoretical $\mathrm{H} \beta / \mathrm{H} \gamma$ ratio is 2.15 (Osterbrock \& Ferland 2006). The Cardelli extinction law was used to derive $A_{\mathrm{V}}$ from the observed $\mathrm{H} \beta / \mathrm{H} \gamma$ value.

\subsection{Ionized gas and stellar kinematics}

We studied the kinematics of the ionized gas using the [O III] $\lambda 5007$ emission-line, except for III Zw 102, for which we used $\mathrm{H} \beta$, which is much stronger than [O III]. Owing to the low spectral resolution of our data (about $5 \AA$ FWHM), no reliable velocity dispersion measurements could be obtained.

The velocity fields are shown in Fig. 7 (row three); in these maps red and blue colors represent redshifted and blueshifted regions with respect to the recessional velocity of each BCG. To estimate the errors on the velocity data, we made a combined plot (all galaxies together) of the uncertainty on the [O III] $\lambda 5007$ emission-line centroid versus the logarithm of its flux. For $\log$ (flux) $>4$ the error is $\$ 5 \mathrm{~km} \mathrm{~s}^{-1}$, in agreement with the mean uncertainty estimated from the scatter in the velocity maps built on the $5577 \AA$ skyline. The error increases to about $10-15 \mathrm{~km} \mathrm{~s}^{-1}$ at $\log$ (flux) $=3$, to $25-30 \mathrm{~km} \mathrm{~s}^{-1}$ at $\log ($ flux $)=2$, and reaches or exceeds $40 \mathrm{~km} \mathrm{~s}^{-1}$ at fainter flux levels.

In addition to the best-fitting stellar template, the pPXF program provides the radial velocity and associated uncertainty for each fitted spaxel, allowing us to produce the galaxy's stellar velocity field (given in Fig. 7, row four).

The uncertainty on the stellar radial velocity varies between $\simeq 10 \mathrm{~km} \mathrm{~s}^{-1}$ in the nuclear regions and $\simeq 40 \mathrm{~km} \mathrm{~s}^{-1}$ in the outer spaxels.

\subsection{Integrated spectroscopy}

For every galaxy we also produced the integrated and nuclear spectrum. To generate the integrated spectrum, we included only those spaxels in which the stellar contribution was successfully modeled as a sum of templates (see Sect. 2.4); in this way we ensured a better correction for the contribution of the underlying stellar population and, therefore, a more accurate determination of the emission-lines fluxes. The integrated spectrum was produced by summing the individual pure emission-line spectra of the selected spaxels. A corresponding continuum spectrum was obtained by summing the best-fit continuum spectra for the same spaxels.

In the same way, we also obtained line-emission and best-fit continuum spectra for the central region by summing over 6-8 spaxels around the continuum peak (for short, we refer to them as nuclear spectra).

Figure 8 displays the integrated spectra for the sample objects. All objects but III Zw 102 display a typical BCG spectrum, in which strong optical emission-lines are superposed on an almost flat continuum. In contrast, III Zw 102's spectral shape,

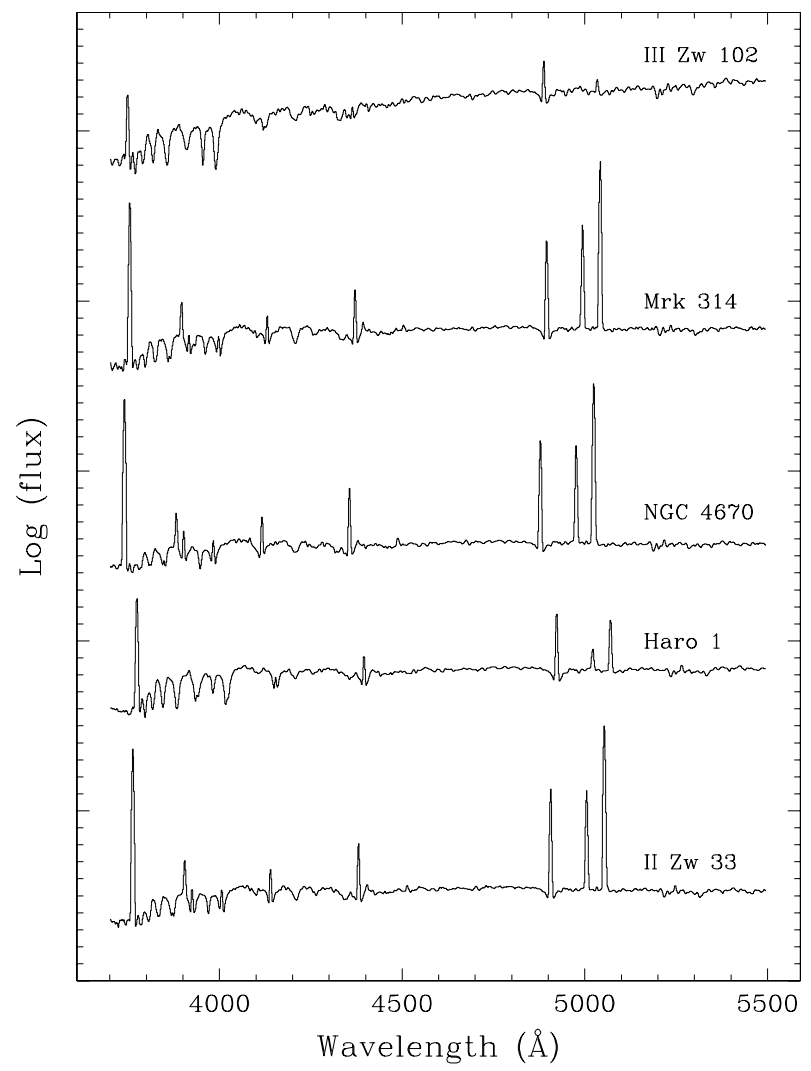

Fig. 8. Integrated spectra of the sample galaxies, obtained by summing the spectra of all spaxels in which the stellar continuum could be fitted by a template (see Sect. 2.4). Spectra are plotted in logarithmic flux scale and were shifted vertically. Major tick-marks on the $y$ axis are spaced 1 dex apart. Nuclear spectra do not exhibit significantly distinct features from the integrated spectra and are not shown.

with a high and red continuum, resembles those typical of an early-type galaxy more than those of BCGs. All objects display prominent absorption features, including the higher-order Balmer lines, $\mathrm{H} \delta, \mathrm{H} \gamma, \mathrm{H} \beta$, the $\mathrm{Ca}$ II $\mathrm{H}$ and $\mathrm{K}$ lines, and the G-band at $4304 \AA$.

For the integrated and nuclear spectra, the flux of the visible emission-lines was measured on the pure emission-line spectrum using the Gaussian profile fitting option in the IRAF task splot. To compute equivalent widths, the continuum level was measured on the best-fit continuum spectrum. For Balmer lines, the continuum level was determined by interpolating over the absorption feature using two continuum windows on either side. The interstellar extinction coefficient, $C(\mathrm{H} \beta)$, was computed through a least-squares fit to the Balmer decrement, using the $\mathrm{H} \beta, \mathrm{H} \delta$ and $\mathrm{H} \gamma$ lines.

Reddening-corrected intensity ratios and equivalent widths of the integrated and nuclear spectra are listed in Table 3. The listed uncertainties on the line ratios and the $\mathrm{H} \beta$ fluxes were derived by summing in quadrature measurement errors, uncertainties on the flux calibration (Sect. 2.3), and the uncertainty on the computed $C(\mathrm{H} \beta)$.

\subsubsection{Heavy-element abundances}

In the two galaxies Mrk 314 and II $\mathrm{Zw} \mathrm{33,} \mathrm{where} \mathrm{the}$ [O III] $\lambda 4363$ auroral line could be measured, electron temperatures and oxygen and neon abundances were derived using the direct method. 
Table 3. Reddening-corrected line ratios for the sample of galaxies normalized to $\mathrm{H} \beta$.

\begin{tabular}{|c|c|c|c|c|c|c|c|c|c|}
\hline \multirow[t]{3}{*}{$\lambda$} & \multirow[t]{3}{*}{ Ion } & \multicolumn{4}{|c|}{ II Zw 33} & \multicolumn{4}{|c|}{ Haro 1} \\
\hline & & \multicolumn{2}{|c|}{ Integrated } & \multicolumn{2}{|c|}{ Nucleus } & \multicolumn{2}{|c|}{ Integrated } & \multicolumn{2}{|c|}{ Nucleus } \\
\hline & & $F_{\lambda}$ & $-W_{\lambda}$ & $F_{\lambda}$ & $-W_{\lambda}$ & $F_{\lambda}$ & $-W_{\lambda}$ & $F_{\lambda}$ & $-W_{\lambda}$ \\
\hline 3727 & [O II] & $3.312 \pm 0.284$ & $58.94 \pm 1.43$ & $3.125 \pm 0.254$ & $48.26 \pm 0.70$ & $2.419 \pm 0.217$ & $24.25 \pm 0.32$ & $2.106 \pm 0.190$ & $19.55 \pm 0.15$ \\
\hline 3835 & H9 & $0.055 \pm 0.006$ & $0.80 \pm 0.05$ & $0.041 \pm 0.004$ & $0.50 \pm 0.04$ & - & - & - & - \\
\hline 3868 & [Ne III] & $0.259 \pm 0.018$ & $3.53 \pm 0.06$ & $0.236 \pm 0.015$ & $2.66 \pm 0.04$ & $0.062 \pm 0.006$ & $0.44 \pm 0.02$ & $0.040 \pm 0.004$ & $0.27 \pm 0.01$ \\
\hline 3889 & $\mathrm{HeI}+\mathrm{H} 8$ & $0.166 \pm 0.012$ & $2.39 \pm 0.04$ & $0.168 \pm 0.011$ & $1.95 \pm 0.03$ & $0.132 \pm 0.009$ & $0.94 \pm 0.02$ & $0.126 \pm 0.009$ & $0.85 \pm 0.01$ \\
\hline 3968 & [Ne III] & $0.228 \pm 0.014$ & $3.21 \pm 0.04$ & $0.209 \pm 0.012$ & $2.47 \pm 0.04$ & - & - & - & - \\
\hline 4101 & $\mathrm{H} \delta$ & $0.256 \pm 0.012$ & $3.68 \pm 0.03$ & $0.256 \pm 0.012$ & $3.02 \pm 0.06$ & $0.244 \pm 0.013$ & $1.84 \pm 0.02$ & $0.239 \pm 0.012$ & $1.73 \pm 0.01$ \\
\hline 4340 & $\mathrm{H} \gamma$ & $0.479 \pm 0.017$ & $7.82 \pm 0.07$ & $0.476 \pm 0.015$ & $6.26 \pm 0.06$ & $0.469 \pm 0.018$ & $3.96 \pm 0.01$ & $0.469 \pm 0.018$ & $3.88 \pm 0.01$ \\
\hline 4363 & [O III] & $0.032 \pm 0.003$ & $0.51 \pm 0.03$ & $0.024 \pm 0.002$ & $0.31 \pm 0.03$ & - & - & - & - \\
\hline 4861 & $\mathrm{H} \beta$ & 1.000 & $19.57 \pm 0.13$ & 1.000 & $15.57 \pm 0.09$ & 1.000 & $10.49 \pm 0.05$ & 1.000 & $10.64 \pm 0.02$ \\
\hline 4959 & [O III] & $0.903 \pm 0.023$ & $18.66 \pm 0.10$ & $0.873 \pm 0.022$ & $14.00 \pm 0.06$ & $0.222 \pm 0.006$ & $2.45 \pm 0.02$ & $0.187 \pm 0.006$ & $2.05 \pm 0.01$ \\
\hline \multirow[t]{2}{*}{5007} & [O III] & $2.653 \pm 0.067$ & $57.47 \pm 0.30$ & $2.554 \pm 0.064$ & $42.93 \pm 0.19$ & $0.656 \pm 0.017$ & $7.70 \pm 0.03$ & $0.532 \pm 0.013$ & $6.22 \pm 0.02$ \\
\hline & & \multicolumn{2}{|c|}{$\begin{array}{c}I(\mathrm{H} \beta)=1061.6 \pm 18.5 \\
F(\mathrm{H} \beta)=2090.3 \pm 283.3 \\
C(\mathrm{H} \beta)=0.294 \pm 0.058 \\
A_{\mathrm{V}}=0.630 \pm 0.125 \\
\text { Area: } 739 \square^{\prime \prime}=24.3 \mathrm{kpc}^{2} \\
V_{\text {stars }}=2828.1 \\
V_{[\mathrm{OIII}]}=2827.4\end{array}$} & \multicolumn{2}{|c|}{$\begin{array}{c}I(\mathrm{H} \beta)=489.6 \pm 8.5 \\
F(\mathrm{H} \beta)=760.0 \pm 80.4 \\
C(\mathrm{H} \beta)=0.191 \pm 0.045 \\
A_{\mathrm{V}}=0.409 \pm 0.097 \\
\text { Area: } 138 \square^{\prime \prime}=4.5 \mathrm{kpc}^{2} \\
V_{\text {stars }}=2829.8 \\
V_{[\mathrm{OIII}]}=2828.4\end{array}$} & \multicolumn{2}{|c|}{$\begin{array}{c}I(\mathrm{H} \beta)=4185.9 \pm 72.8 \\
F(\mathrm{H} \beta)=9800.7 \pm 1628.1 \\
C(\mathrm{H} \beta)=0.369 \pm 0.072 \\
A_{\mathrm{V}}=0.791 \pm 0.154 \\
\text { Area: } 1432 \square^{\prime \prime}=94.2 \mathrm{kpc}^{2} \\
V_{\text {stars }}=3772.0 \\
V_{[\mathrm{OIII}]}=3781.5\end{array}$} & \multicolumn{2}{|c|}{$\begin{array}{c}I(\mathrm{H} \beta)=1532.0 \pm 26.5 \\
F(\mathrm{H} \beta)=3600.5 \pm 597.4 \\
C(\mathrm{H} \beta)=0.371 \pm 0.072 \\
A_{\mathrm{V}}=0.794 \pm 0.154 \\
\text { Area: } 108 \square^{\prime \prime}=7.1 \mathrm{kpc}^{2} \\
V_{\text {stars }}=3758.0 \\
V_{[\mathrm{OIII}]}=3759.6\end{array}$} \\
\hline \multirow[t]{3}{*}{$\lambda$} & \multirow[t]{3}{*}{ Ion } & \multicolumn{4}{|c|}{ NGC 4670 } & \multicolumn{4}{|c|}{ Mrk 314} \\
\hline & & \multicolumn{2}{|c|}{ Integrated } & \multicolumn{2}{|c|}{ Nucleus } & \multicolumn{2}{|c|}{ Integrated } & \multicolumn{2}{|c|}{ Nucleus } \\
\hline & & $F_{\lambda}$ & $-W_{\lambda}$ & $F_{\lambda}$ & $-W_{\lambda}$ & $F_{\lambda}$ & $-W_{\lambda}$ & $F_{\lambda}$ & $-W_{\lambda}$ \\
\hline 3727 & [O II] & $3.051 \pm 0.280$ & $60.52 \pm 0.97$ & $2.487 \pm 0.200$ & $44.16 \pm 0.30$ & $3.255 \pm 0.270$ & $50.66 \pm 0.92$ & $2.859 \pm 0.264$ & $54.09 \pm 0.60$ \\
\hline 3835 & H9 & $0.048 \pm 0.004$ & $0.89 \pm 0.03$ & $0.044 \pm 0.004$ & $0.71 \pm 0.01$ & $0.053 \pm 0.005$ & $0.97 \pm 0.07$ & $0.037 \pm 0.003$ & $0.78 \pm 0.02$ \\
\hline 3868 & [Ne III] & $0.202 \pm 0.016$ & $3.32 \pm 0.03$ & $0.208 \pm 0.012$ & $3.37 \pm 0.01$ & $0.296 \pm 0.019$ & $3.58 \pm 0.06$ & $0.297 \pm 0.022$ & $4.24 \pm 0.02$ \\
\hline 3889 & $\mathrm{HeI}+\mathrm{H} 8$ & $0.187 \pm 0.014$ & $3.18 \pm 0.02$ & $0.182 \pm 0.011$ & $2.84 \pm 0.01$ & $0.165 \pm 0.010$ & $2.91 \pm 0.08$ & $0.148 \pm 0.010$ & $2.12 \pm 0.02$ \\
\hline 3968 & [Ne III] & $0.217 \pm 0.013$ & $3.79 \pm 0.02$ & $0.215 \pm 0.010$ & $3.55 \pm 0.01$ & $0.245 \pm 0.013$ & $3.34 \pm 0.03$ & $0.214 \pm 0.014$ & $3.07 \pm 0.01$ \\
\hline 4101 & $\mathrm{H} \delta$ & $0.263 \pm 0.014$ & $4.52 \pm 0.04$ & $0.259 \pm 0.013$ & $4.53 \pm 0.02$ & $0.256 \pm 0.011$ & $3.29 \pm 0.03$ & $0.241 \pm 0.012$ & $3.40 \pm 0.01$ \\
\hline 4340 & $\mathrm{H} \gamma$ & $0.458 \pm 0.016$ & $8.99 \pm 0.04$ & $0.468 \pm 0.013$ & $9.71 \pm 0.04$ & $0.477 \pm 0.015$ & $6.85 \pm 0.05$ & $0.469 \pm 0.017$ & $7.88 \pm 0.04$ \\
\hline 4363 & [O III] & - & - & - & - & $0.037 \pm 0.002$ & $0.53 \pm 0.02$ & $0.038 \pm 0.002$ & $0.65 \pm 0.02$ \\
\hline 4861 & $\mathrm{H} \beta$ & 1.000 & $21.93 \pm 0.09$ & 1.000 & $26.03 \pm 0.14$ & 1.000 & $17.29 \pm 0.16$ & 1.000 & $20.41 \pm 0.11$ \\
\hline 4959 & [O III] & $0.765 \pm 0.019$ & $17.89 \pm 0.08$ & $0.868 \pm 0.021$ & $24.11 \pm 0.08$ & $1.070 \pm 0.026$ & $19.51 \pm 0.18$ & $1.175 \pm 0.029$ & $25.34 \pm 0.20$ \\
\hline \multirow[t]{2}{*}{5007} & [O III] & $2.223 \pm 0.056$ & $54.01 \pm 0.23$ & $2.500 \pm 0.061$ & $72.26 \pm 0.23$ & $3.053 \pm 0.075$ & $59.08 \pm 0.55$ & $3.354 \pm 0.085$ & $77.07 \pm 0.63$ \\
\hline & & $\begin{array}{r}I(\mathrm{H} \beta)=66 \\
F(\mathrm{H} \beta)=104 \\
C(\mathrm{H} \beta)=0 . \\
A_{\mathrm{V}}=0.4 \\
\text { Area: } 2571 \\
V_{\text {stars }}= \\
V_{[\mathrm{OIII}]}\end{array}$ & $\begin{array}{l}0 \pm 116.1 \\
.3 \pm 1598.2 \\
9 \pm 0.066 \\
\pm 0.141 \\
=30.3 \mathrm{kpc}^{2} \\
026.2 \\
049.7\end{array}$ & $\begin{array}{r}I(\mathrm{H} \beta)=31 \\
F(\mathrm{H} \beta)=55 \\
C(\mathrm{H} \beta)=0 . \\
A_{\mathrm{V}}=0.5 \\
\text { Area: } 92 \square^{\prime} \\
V_{\text {stars }}= \\
V_{[\mathrm{OIII}]}\end{array}$ & $\begin{array}{l}1.8 \pm 54.3 \\
.2 \pm 382.6 \\
4 \pm 0.029 \\
\pm 0.062 \\
=1.1 \mathrm{kpc}^{2} \\
019.9 \\
1051.1\end{array}$ & $\begin{array}{r}I(\mathrm{H} \beta)=14 \\
F(\mathrm{H} \beta)=36 \\
C(\mathrm{H} \beta)=0 \\
A_{\mathrm{V}}=0.8 \\
\text { Area: } 739 \square \\
V_{\text {stars }}= \\
V_{[\text {OIII] }]}\end{array}$ & $\begin{array}{l}1.8 \pm 26.2 \\
1.7 \pm 394.0 \\
34 \pm 0.047 \\
\pm 0.100 \\
=14.6 \mathrm{kpc}^{2} \\
067.6 \\
2076.3\end{array}$ & $\begin{array}{r}I(\mathrm{H} \beta)=99 \\
F(\mathrm{H} \beta)=19 \\
C(\mathrm{H} \beta)=0 . \\
A_{\mathrm{V}}=0.64 \\
\text { Area: } 108 \text { व } \\
V_{\text {stars }}= \\
V_{[\mathrm{OIII}]}=\end{array}$ & $\begin{array}{l}0 \pm 17.3 \\
.5 \pm 330.3 \\
9 \pm 0.072 \\
\pm 0.155 \\
=2.1 \mathrm{kpc}^{2} \\
064.6 \\
2080.3\end{array}$ \\
\hline$\lambda$ & Ion & & III Z & 102 & & & & & \\
\hline & & $F_{\lambda}{ }^{\text {Integ }}$ & ted $-W_{\lambda}$ & $F_{\lambda}$ & $-W_{\lambda}$ & & & & \\
\hline 3727 & {$[\mathrm{O} \mathrm{II}]$} & $1.618 \pm 0.155$ & $9.81 \pm 0.20$ & $1.269 \pm 0.120$ & $11.38 \pm 0.11$ & & & & \\
\hline 3835 & H9 & - & - & - & - & & & & \\
\hline 3868 & [Ne III] & - & - & - & - & & & & \\
\hline 3889 & $\mathrm{HeI}+\mathrm{H} 8$ & - & - & - & - & & & & \\
\hline 3968 & [Ne III] & - & - & - & - & & & & \\
\hline 4101 & $\mathrm{H} \delta$ & $0.237 \pm 0.015$ & $1.01 \pm 0.01$ & $0.233 \pm 0.012$ & $1.52 \pm 0.02$ & & & & \\
\hline 4340 & $\mathrm{H} \gamma$ & $0.469 \pm 0.019$ & $2.20 \pm 0.02$ & $0.469 \pm 0.018$ & $3.45 \pm 0.03$ & & & & \\
\hline 4363 & [O III] & - & - & - & - & & & & \\
\hline 4861 & $\mathrm{H} \beta$ & 1.000 & $4.93 \pm 0.02$ & 1.000 & $8.53 \pm 0.04$ & & & & \\
\hline 4959 & [O III] & $0.112 \pm 0.004$ & $0.58 \pm 0.01$ & $0.086 \pm 0.003$ & $0.76 \pm 0.01$ & & & & \\
\hline 5007 & [O III] & $0.319 \pm 0.008$ & $1.77 \pm 0.01$ & $0.230 \pm 0.006$ & $2.18 \pm 0.01$ & & & & \\
\hline & & $\begin{array}{r}I(\mathrm{H} \beta)=16 \\
F(\mathrm{H} \beta)=603 \\
C(\mathrm{H} \beta)=0 . \\
A_{\mathrm{V}}=1.2 \\
\text { Area: } 2171 \square \\
V_{\text {stars }}= \\
V_{[\mathrm{OIII}]}\end{array}$ & $\begin{array}{l}2.9 \pm 29.2 \\
4 \pm 1075.0 \\
2 \pm 0.077 \\
\pm 0.165 \\
=27.2 \mathrm{kpc}^{2} \\
611.7 \\
615.6\end{array}$ & $\begin{array}{r}I(\mathrm{H} \beta)=8 \\
F(\mathrm{H} \beta)=36 \\
C(\mathrm{H} \beta)=0 \\
A_{\mathrm{V}}=1.3 \\
\text { Area: } 123 \\
V_{\text {stars }} \\
V_{[\mathrm{OIII}]}\end{array}$ & $\begin{array}{l}.2 \pm 14.2 \\
7.1 \pm 628.9 \\
54 \pm 0.075 \\
\pm 0.160 \\
=1.5 \mathrm{kpc}^{2} \\
621.9 \\
1608.7\end{array}$ & & & & \\
\hline
\end{tabular}

Notes. Reddening-corrected line fluxes normalized to $F(\mathrm{H} \beta)=1$. The observed $\mathrm{H} \beta$ flux, $I(\mathrm{H} \beta)$, the reddening-corrected $\mathrm{H} \beta$ flux, $F(\mathrm{H} \beta)$ (both $\times 10^{-16} \mathrm{erg} \mathrm{cm}^{-2} \mathrm{~s}^{-1}$ ), the reddening coefficient, $C(\mathrm{H} \beta)$, and $A_{\mathrm{V}}$ (derived as $2.14 \times C(\mathrm{H} \beta)$ ) are listed for each region. Also shown are the area covered by the integrated spectra and the corresponding (luminosity weighted) average stellar and gas velocities. The quoted uncertainties account for measurement, flux-calibration, and reddening coefficient errors.

First, we computed the electron temperature $T_{\mathrm{e}}(\mathrm{O}$ III $)$ from the [O III] $\lambda 4363 /[\mathrm{O}$ III] $\lambda 4959+\lambda 5007$ ratio using the equations provided by Aller (1984). Because there are no useful lines to obtain the electron density, $N_{\mathrm{e}}$, in our spectra we assumed $N_{\mathrm{e}}=100 \mathrm{~cm}^{-3}$.

The metal abundances were derived using the revised expressions in Izotov et al. (2006b), which take into account the latest atomic data and incorporate an appropriate grid of photoionization models with state-of-the-art model atmospheres. We used their Eqs. (3) and (5) to compute the $\mathrm{O}^{+} / \mathrm{H}, \mathrm{O}^{++} / \mathrm{H}$ and $\mathrm{Ne}^{2+}$ abundances, after computing $T_{\mathrm{e}}(\mathrm{O}$ II) using Eq. (14). The total oxygen abundance was obtained by simply summing the abundances of $\mathrm{O}^{+}$and $\mathrm{O}^{++}$, because the fraction of the $\mathrm{O}^{+++}$ion is generally negligible. The correction factor 
Table 4. Electron temperatures and heavy-metal abundances.

\begin{tabular}{|c|c|c|c|c|c|c|c|c|c|c|}
\hline \multirow[b]{2}{*}{ Value } & \multicolumn{2}{|c|}{ II Zw 33} & \multicolumn{2}{|c|}{ Haro 1} & \multicolumn{2}{|c|}{ NGC 4670} & \multicolumn{2}{|c|}{ Mrk 314} & \multicolumn{2}{|c|}{ III Zw 102} \\
\hline & Integrated & Nuclear & Integrated & Nuclear & Integrated & Nuclear & Integrated & Nuclear & Integrated & Nuclear \\
\hline$T T_{\mathrm{e}}(\mathrm{O}$ III) $(\mathrm{K})$ & $12290 \pm 330$ & $11270 \pm 360$ & - & - & - & - & $12330 \pm 250$ & $12090 \pm 210$ & - & - \\
\hline$T_{\mathrm{e}}(\mathrm{O}$ II $)(\mathrm{K})$ & $12080 \pm 290$ & $11160 \pm 350$ & - & - & - & - & $12110 \pm 210$ & $11910 \pm 190$ & - & - \\
\hline $12+\log (\mathrm{O} / \mathrm{H})^{1}$ & $8.06 \pm 0.03$ & $8.16 \pm 0.04$ & - & - & & & $8.08 \pm 0.03$ & $8.10 \pm 0.03$ & & \\
\hline $12+\log (\mathrm{O} / \mathrm{H})^{2}$ & - & - & 8.36 & 8.40 & 8.29 & 8.37 & - & - & 8.44 & 8.49 \\
\hline $\log (\mathrm{Ne} / \mathrm{O})$ & $-0.85 \pm 0.05$ & $-0.85 \pm 0.06$ & - & - & - & - & $-0.83 \pm 0.04$ & $-0.82 \pm 0.04$ & - & - \\
\hline
\end{tabular}

Notes. ${ }^{(1)}$ Oxygen abundances computed using the $T_{\mathrm{e}}$ method. ${ }^{(2)}$ Oxygen abundances derived from Pilyugin \& Thuan (2005); uncertainties are about 0.1 dex.

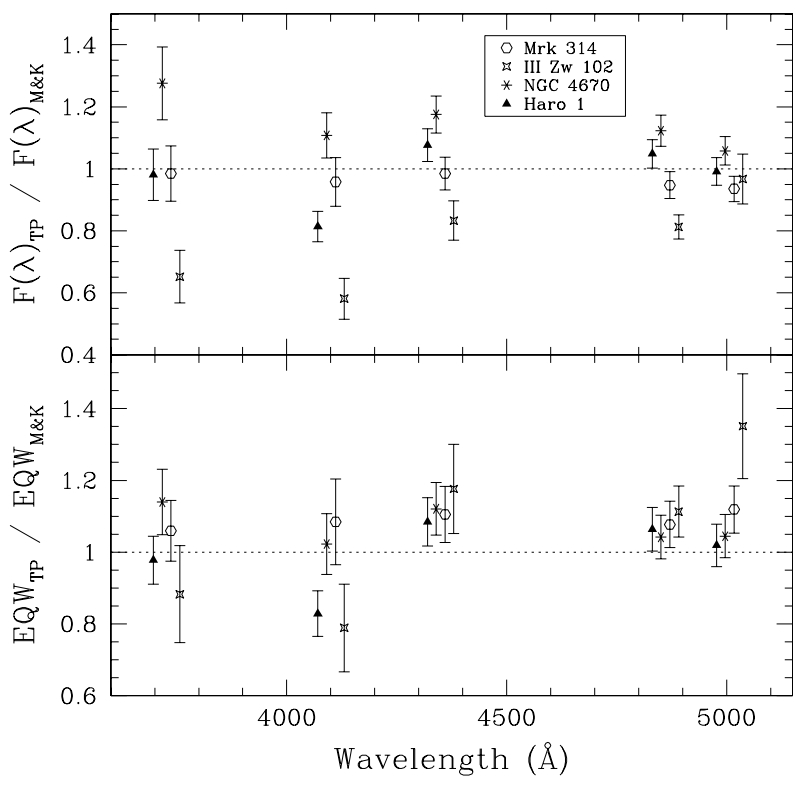

Fig. 9. Upper panel: ratio between the fluxes computed by direct summation of our integrated spectra, after correction for foreground galactic extinction, and the (galactic-extinction-corrected) integrated fluxes published by Moustakas \& Kennicutt (2006). Lower panel: ratio between equivalent width measurements. To avoid overlapping of error bars (which include the uncertainties on both datasets), datapoints were slightly shifted from their nominal position on the $X$ axis.

for $\mathrm{Ne}, I C F\left(\mathrm{Ne}^{2+}\right)$, was computed using Eq. (19). (Using the $\mathrm{Ne}^{++} / \mathrm{O}^{++}$ratio instead of the ICF would yield an Ne abundance about 0.2 dex higher.)

For the remaining three galaxies, where [O III] $\lambda 4363$ could not be measured, we had to rely on the so-called strong-line methods to derive the oxygen abundance. We adopted the revised calibration of the P-method (Pilyugin 2000, 2001), provided in Pilyugin \& Thuan (2005).

Electron temperatures and abundances are listed in Table 4. For II Zw 33 and Mrk 314, we provide only the abundances derived from the direct method; since both galaxies lie in the transition zone $8.0 \leq 12+\log (\mathrm{O} / \mathrm{H}) \leq 8.3$, the Pilyugin \& Thuan (2005) calibration does not give reliable abundance values.

\subsubsection{Comparison with literature data}

To assess the quality of our measurements, we compared our integrated fluxes and equivalent widths with the integrated data published by Moustakas \& Kennicutt (2006). Four of our objects are in common with their sample, the exception is II Zw 33.

For this comparison, our measurements were corrected for foreground galactic extinction only. The results of the comparison are displayed in Fig. 9. Taking into account the different integration area used in the Moustakas \& Kennicutt (2006) spectra (a $30^{\prime \prime} \times 50^{\prime \prime}$ aperture) and our own spectra, the comparison looks fair.

\section{Discussion}

\subsection{Zw 33 (=Mrk 1094)}

II Zw 33 is a well-studied object, cataloged as BCG in Thuan \& Martin (1981). Optical imaging and surface photometry has been presented in several works (Loose \& Thuan 1986; Cairós et al. 2001a,b; Gil de Paz et al. 2003; Gil de Paz \& Madore 2005). The presence of a central SF bar and the striking twisting of the isophotes have been interpreted as interaction signs (Méndez et al. 1999). II Zw 33 is also a well-known Wolf-Rayet (WR) galaxy, included in the WR galaxy lists by Vacca \& Conti (1992) and Schaerer et al. (1999).

Maps of II Zw 33 are displayed in Figs. 6 and 7. At the II Zw 33 distance, the 4 .' 2 fiber diameter provides a spatial sampling of 760 pc per spaxel.

The line emission is concentrated along a central bar-like structure, with no clear peak, while the stellar emission extends farther out from the central SF regions, evidencing the presence of a much more extended, more evolved stellar population. The continuum map displays a clearly defined peak, located roughly at the center of the galaxy.

The excitation map $([\mathrm{O} \mathrm{III}] / \mathrm{H} \beta)$ traces the regions of star formation, as expected for objects photoionized by stars. The peak in $[\mathrm{O} \mathrm{III]}] / \mathrm{H} \beta$ is located at the southern tip of the bar which is knot "A" as labeled in Méndez et al. (1999); their value of 5.5 agrees well with the peak value we find, $\simeq 4.5$ (the small discrepancy is likely due to the difference in spatial resolution).

The extinction is relatively uniform in the central region, with $A_{\mathrm{V}}$ values around 0.4 ; these values are significantly lower than those published by Méndez et al. (1999).

The [O III] velocity map does not show a clear rotation pattern, although the northwest side seems to have a slightly higher radial velocity as a whole. The ionized gas kinematics is in good qualitative agreement with the H I kinematical map published by Walter et al. (1997, see their Fig. 6). The stellar velocity field is not as extended as required to make a meaningful comparison, and is also much noisier.

We derived an oxygen abundance of $12+\log (\mathrm{O} / \mathrm{H})=8.06$, in good agreement with the value of $12+\log (\mathrm{O} / \mathrm{H})=7.99$ reported by Shi et al. (2005), who also used the $T_{\mathrm{e}}$ method.

The Wolf-Rayet bump around 4600-4680 $\AA$ is visible in two spaxels, marked by a star in the [O III] flux maps in Fig. 6.

\subsection{Haro 1 (= NGC 2415)}

Haro 1 is classified as BCG by Gordon \& Gottesman (1981) and Klein et al. (1984), and is included in the H II galaxy sample studied by Deeg et al. (1997). It has been defined as a paired 
galaxy with little (if any) tidal disturbance (Schneider \& Salpeter 1992). Its companion is UGC 3937, with a redshift difference of only $210 \mathrm{~km} \mathrm{~s}^{-1}$ and a projected distance of $350 \mathrm{kpc}$ (23 arcmin at Haro 1's distance). Broad-band surface brightness photometry was presented in Cairós et al. (2001a,b).

Maps are shown in Figs. 6 and 7 (Col. 2). At the Haro 1 distance, the spatial resolution is about $1.1 \mathrm{kpc}$ per spaxel.

Haro 1 displays outer regular isophotes in the continuum maps. (The jagged appearance is the result of masking several foreground stars; for the sake of clarity, a smaller star, located at $\approx 30^{\prime \prime}$ east from the center, has been left, as well as the background galaxy on the west-side). The continuum emission peak is placed roughly at the center of the isophotes. The emission-line maps display a more irregular pattern: a considerably large filament extends to the northwest, and the emission peak seems to be slightly offset to the west with respect to the continuum peak.

The $[\mathrm{O} \mathrm{III}] / \mathrm{H} \beta$ ratio map has a shallow minimum, 0.40 , in the galaxy's central region (where the emission peaks), and increases slightly outward, reaching its maximum at the position of the extension detected in emission-line maps. Radial changes in the $[\mathrm{O} \mathrm{III}] / \mathrm{H} \beta$ ratio have been found in spiral galaxies, and have been interpreted as variation in the heavy element abundances (Searle 1971; Smith 1975). Interestingly, Haro 1 is the most luminous object in our sample, and it has also been classified as an Sm galaxy (HyperLeda ${ }^{5}$; Paturel et al. 2003).

The extinction map shows little or no spatial variations, with an average extinction $A_{\mathrm{V}} \sim 0.90$.

Haro 1's kinematics show an overall regular rotation about an axis oriented southeast-northwest, with nearly the same pattern and amplitude (about $160 \mathrm{~km} \mathrm{~s}^{-1}$ ) both in the gas and in the stellar velocity maps.

The value derived for the metallicity, $12+\log (\mathrm{O} / \mathrm{H})=8.36$, is slightly lower that the value of $12+\log (\mathrm{O} / \mathrm{H})=8.52$ derived in Shi et al. (2005), who used the P-method (as calibrated in Pilyugin 2001).

\section{3. $N G C 4670$ (= Haro 9, Arp 163)}

NGC 4670 has been classified as a blue amorphous galaxy (Hunter et al. 1994; Marlowe et al. 1997), as a peculiar, barred S0/a galaxy (de Vaucouleurs et al. 1991), and as a BCG (Gil de Paz et al. 2003). It is listed as a peculiar galaxy with "diffuse counter tails" in Arp's catalog (Arp 1966), and as a WR galaxy in Mas-Hesse \& Kunth (1999) and Schaerer et al. (1999). Broad-band photometry and $\mathrm{H} \alpha$ imaging have been provided in Gil de Paz et al. (2003), Gil de Paz \& Madore (2005) and Marlowe et al. (1997).

Maps are shown in Figs. 6 and 7 (Col. 3). At the NGC 4670 distance, the VIRUS-P IFU 4".2 fiber diameter corresponds to about 460 pc.

The continuum map shows an outer regular morphology, with elliptical isophotes; the bulge, extremely bright, is clearly elongated on an east-west axis. The continuum peak appears to be slightly displaced to the east, in agreement with the $B$-band image (Fig. 1). In emission-lines, the galaxy displays quite a complex pattern, with a central, roughly circular and very bright H II region surrounded by an extended filamentary envelope, which has several bubbles, loops, and extensions. Remarkable are the two biggest filaments, which expand to the southwest and northeast, extending to about 7.5 and $6.5 \mathrm{kpc}$, respectively.

\footnotetext{
5 http://leda.univ-lyon1.fr
}

The ionization map displays an intriguing structure. The $[\mathrm{O} \mathrm{III}] / \mathrm{H} \beta$ ratio clearly shows higher values in the central region, with a peak value $\sim 3$, and at the outer edge of the two larger extensions. Highest values of $[\mathrm{O} \mathrm{III}] / \mathrm{H} \beta$ in the central knot, where the line-emission intensity peaks as well, are consistent with photoionization produced by hot stars; it is not clear, however, which ionizing mechanism acts on the filaments. Although the morphology suggests a shock-like excitation mechanism, no conclusions can be drawn without measuring additional diagnostic lines. The excitation values we found agree very well with those reported in Hunter et al. (1994), [O III]/H $\beta=2.97$, derived for the spectra positioned at the center of the galaxy.

The extinction map shows a roughly flat behavior in the inner region, with $A_{\mathrm{V}}$ around 0.40 , and seems to be dominated by noise in the outer parts.

Hunter et al. (1994), analyzing VLA data, reported an "ordered rotation east-west, and the motion appears to be that of a disk at the same position angle as the optical galaxy ( -100 deg)". Our velocity maps show a more complex picture. At first sight a rotation pattern, though somewhat irregular, is visible in the gaseous component, with an amplitude of about $150 \mathrm{~km} \mathrm{~s}^{-1}$, around an axis oriented roughly northwestsoutheast; the velocity gradient seems to be slightly steeper on the northeast side of the galaxy compared to the southwest part. The stellar velocity field is overall flat, and broadly agrees with the gas kinematics in the same area, except for the slight velocity rise toward its east edge, visible in the [O III] radial velocity map, but not in the stellar one.

The behavior of the gas and stellar kinematics can be better perceived in Fig. 10, where we plot a velocity profile along a line with PA $=50 \mathrm{deg}$, where the gas distribution extends farthest from the galaxy center, and a velocity profile at PA $=-100 \mathrm{deg}$, the value adopted by Hunter et al. (1994). Both graphs show a somewhat irregular rise of the gas velocity in a central region of about $40^{\prime \prime}$ in size, and are flat, though noisier, outside it. The stellar velocity profile shows that the stars co-rotate with the gas, but with a lower velocity gradient, and seems to flatten out at a somewhat smaller radial distance than the gas. Furthermore, the isovelocity contour at $V=1050 \mathrm{~km} \mathrm{~s}^{-1}$ in the [O III] velocity field is clearly S-shaped, a characteristic typical of barred galaxies (Peterson et al. 1978).

Thus, the kinematics of NGC 4670 is unlike that of a rotating disk, and more resembles the kinematics of a barred galaxy, in line with its morphological classification, $\mathrm{SB}(\mathrm{s}) 0 / \mathrm{aP}$ ?, listed in de Vaucouleurs et al. (1991).

The derived metallicity, $12+\log (\mathrm{O} / \mathrm{H})=8.29$, is slightly lower but consistent with the value of $12+\log (\mathrm{O} / \mathrm{H})=8.4 \mathrm{re}$ ported in Mas-Hesse \& Kunth (1999).

The Wolf-Rayet bump around 4600-4680 $\AA$ is visible in three spaxels, marked by a star in the [O III] flux maps in Fig. 6.

\subsection{Mrk 314 (= NGC 7468)}

Mrk 314 has been cataloged as a polar-ring galaxy candidate (Whitmore et al. 1990; van Driel et al. 2000) and is included in the Mazzarella \& Boroson (1993) catalog of multiple-nuclei galaxies. Surface photometry in the optical and in the NIR was presented in Cairós et al. (2001a,b), Caon et al. (2005), and Noeske et al. (2005).

The maps of Mrk 314 are shown in Figs. 6 and 7 (Col. 4). The spatial sampling is about 590 pc per spaxel.

The galaxy displays a similar morphology in both emissionline and continuum maps, with the SF knots aligned along the northeast-southwest direction. In the continuum map, a more 


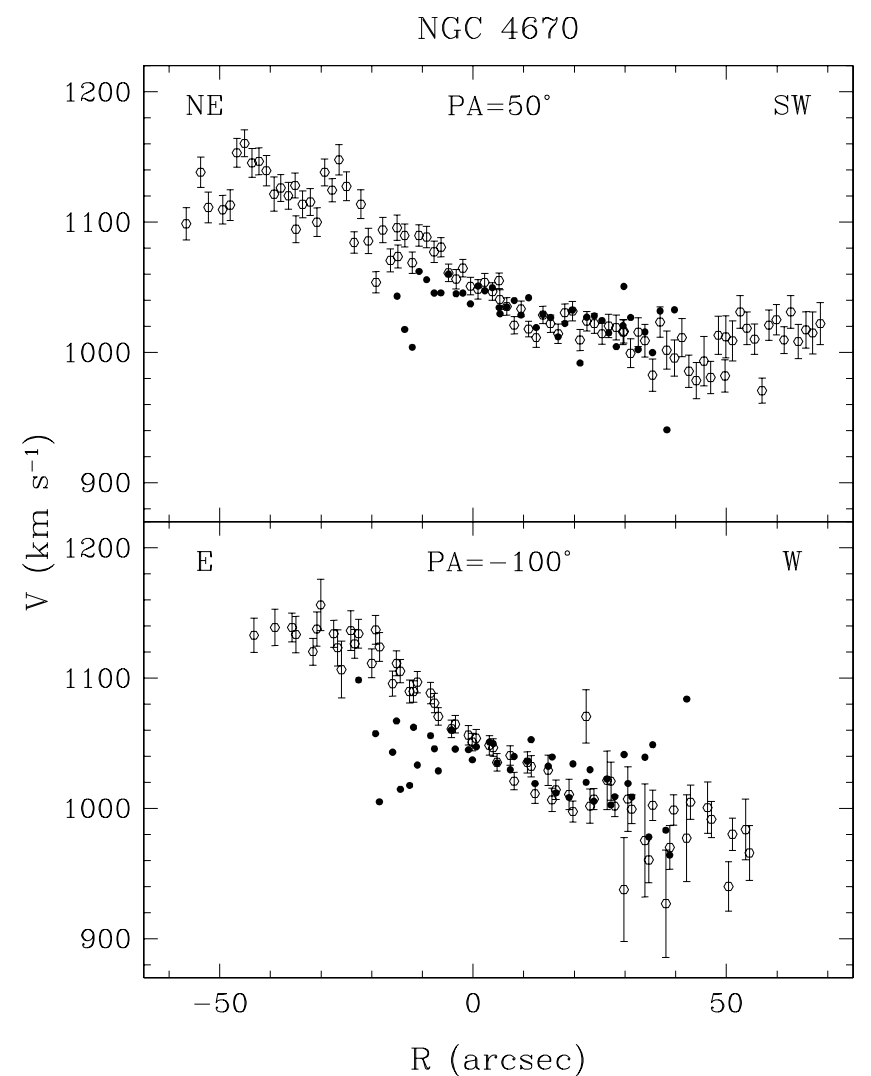

Fig. 10. Upper panel: kinematical profile of NGC 4670 along a spatial cut through the reference point $(0.0,8.0)$ and $\mathrm{PA}=50 \mathrm{deg}$. The $x$ axis is the distance of the projection of each spaxel on the above line to the reference point; only spaxels whose distance to the line is less than $6^{\prime \prime}$ were selected and plotted. Lower panel: kinematical profile through the same point as above and $\mathrm{PA}=-100 \mathrm{deg}$. Open points are [O III] velocities, solid points are stellar velocities.

extended stellar component, with elliptical isophotes, surrounds the SF region. The brighter knot is located roughly at the center of the outer isophotes, and a tail-like feature departs in the southwest direction; a second filament extends out to the northwest. In the emission-line maps, the emission is concentrated in a central SF bar, the emission peak being slightly displaced from the continuum peak.

The peak of the $[\mathrm{O} \mathrm{III}] / \mathrm{H} \beta$ ratio is spatially co-located with the line emission maximum, as expected for objects ionized by OB hot stars; the excitation peak value of $\simeq 4.0$ agrees well with the value found by García-Lorenzo et al. (2008).

The extinction map does not show any definite pattern, but a more or less homogeneous distribution with somewhat higher values at the south, and a $A_{\mathrm{V}}$ value around $0.7-0.8$ in the central part; this result is consistent with the $\mathrm{H} \alpha / \mathrm{H} \beta$ map presented in García-Lorenzo et al. (2008), measured in a smaller FOV $\left(16^{\prime \prime} \times 12^{\prime \prime}\right)$.

The ionized gas velocity field displays a complicated pattern. Around the main body of the galaxy, the velocity field is similar to a rotating disk, with the rotation axis oriented east-west, the northern side approaching and the southern side receding. This rotation is perturbed in the central galaxy regions (within 10"), however, where the velocity field shows an S-shape distortion, indicative of the presence of a second rotating system. South of the main body (this area coincides with the tail-like feature), the ionized gas is not rotating with the main-body galaxy disk.

An extensively kinematic analysis of this galaxy has been performed by Shalyapina et al. (2004), who found that Mrk 314 harbors two kinematics subsystems of ionized gas: the main gaseous disk, and a smaller inner disk oriented orthogonally to it. Our coarse spatial resolution does not allow us to either confirm or disprove the existence of this inner disk, but the kinematics of the main disk agree well with the data shown by Shalyapina et al. (2004): see for instance the peak in the velocity profile at $\simeq 30^{\prime \prime}$ south of the nucleus, and then the sharp decrease (their Fig. 2a).

The stellar velocity field looks more irregular, though there seems to be some rotation along an axis at about PA $\sim 45 \mathrm{deg}$, with a total amplitude of about $70-80 \mathrm{~km} \mathrm{~s}^{-1}$.

The oxygen abundance we found in the integrated spectrum, $12+\log (\mathrm{O} / \mathrm{H})=8.08$, is somewhat lower than that reported by Shi et al. (2005), $12+\log (\mathrm{O} / \mathrm{H})=8.26$ (also derived using the $T_{\mathrm{e}}$ method), and those reported by García-Lorenzo et al. (2008), which range spatially from 8.14 to 8.35 (using the empirical calibrator N2, Denicoló et al. 2002).

\subsection{Zw 102 (=NGC 7625, Arp 212)}

III Zw 102 is a galaxy thoroughly studied in a wide range of wavelengths, but still a puzzling system. It is included in the Vorontsov-Velyaminov catalog of interacting galaxies (Vorontsov-Velyaminov 1959) and in Arp's Atlas of Peculiar Galaxies (Arp 1966). It has been classified as an SA(rs) a pec in RC2 (de Vaucouleurs et al. 1976), as an S0 pec (Lynds \& Furenlid 1973), as an elliptical with dust lanes (Hawarden et al. 1981; Bertola 1987; Yasuda et al. 1992), and as a BCG (Thuan \& Martin 1981). Whitmore et al. (1990) considered III Zw 102 to be "related to polar-ring galaxies". Broad-band photometry was presented in Cairós et al. (2001a,b). III Zw 102 kinematics was first studied by Demoulin (1969), who concluded from optical spectroscopy that the galaxy rotates as a nearly solid body within the central $30^{\prime \prime}$. H I gas displays a more complicated behavior, with the outer H I showing apparent counter-rotation with respect to the stellar and molecular gas components ( $\mathrm{Li}$ et al. 1993).

Results from 3D investigations of III Zw 102 have been published in García-Lorenzo et al. (2008), where the central $33.6^{\prime \prime} \times 29.4^{\prime \prime}$ of the galaxy were mapped with a spatial resolution of $2.7^{\prime \prime}$ spaxel $^{-1}$, using the IFS INTEGRAL, and in Moiseev (2008), where the distribution and kinematics of the ionized gas was studied by means of Fabry-Perot scanning interferometry.

Maps of III Zw 102 are shown in Figs. 6 and 7 (Col. 5). The VIRUS-P IFU 4". 2 fiber diameter corresponds to about $470 \mathrm{pc}$.

In the continuum the galaxy displays outer circular isophotes, indicative of the presence of an underlying population of older stars, extending up to a radius of about $7 \mathrm{kpc}$. The central regions are more irregular and have elliptical isophotes, with the major axis oriented in the northeast-southwest direction. Dust is clearly seen on the continuum map: a dust lane crosses the galaxy in a southwest-northeast direction, almost parallel to the ellipses' major axis, and large dust patches are also evident at the south, almost parallel to the galaxy minor axis. In the narrowband maps the morphology is more irregular, with several filaments, bubbles, and holes. The extended filaments, departing to the southeast and to the northwest, are very well traced in the $[\mathrm{O}$ II] and $\mathrm{H} \beta$ maps.

The $[\mathrm{O} \mathrm{III}] / \mathrm{H} \beta$ ratio (see Fig. 7) is lower in the central part of the galaxy, with a minimum value of $\simeq 0.22$, in good agreement with the values published by García-Lorenzo et al. (2008). The excitation, like in Haro 1, increases toward the galaxy outskirts, a behavior often seen in spiral galaxies (Smith 1975). Just like 
Haro 1, III Zw 102 is a luminous object, which has been classified as a spiral by de Vaucouleurs et al. (1976).

The extinction map is highly inhomogeneous and traces the dust lanes detected in optical frames and color maps well. The values indicate a considerable amount of dust.

The stellar kinematics display an overall rotation around an axis oriented approximately northwest-southeast, with the northeast side approaching and the southwest receding, and a velocity amplitude of about $160 \mathrm{~km} \mathrm{~s}^{-1}$. The gas kinematics, which in this galaxy is better mapped by the $\mathrm{H} \beta$ line (stronger than [O III]) broadly agrees with the stellar kinematics in the inner region. Notable are the two features protruding from either side of the galaxy, and which, together with the knot $40^{\prime \prime}$ northeast of the galaxy center, seem to form a structurally and kinematically independent system. These findings agree with the results of the detailed analysis of this galaxy published by Moiseev (2008): III Zw 102 consists of two distinct systems: an inner disk, where gas and stars co-rotate, and a polar ring orthogonal to it.

The oxygen abundances, $12+\log (\mathrm{O} / \mathrm{H})=8.44$ in the integrated spectrum, and $12+\log (\mathrm{O} / \mathrm{H}=8.49$ in the nuclear spectrum, are much lower than those derived in García-Lorenzo et al. (2008) using the empirical calibrator N2 (Denicoló et al. 2002), ranging from 8.81 to 8.84 in the circumnuclear regions and about 8.85 for the nucleus. While the disagreement can be due partly to the different corrections for the underlying absorption at $\mathrm{H} \beta$, discrepancies of up to 0.4 dex between values obtained with the P-method and N2 have already been reported (Shi et al. 2005).

\section{Summary}

We presented results of an IFS analysis of five luminous BCG galaxies, based on VIRUS-P data covering the wavelength interval 3550-5850 $\AA$ with a resolution of about $5 \AA$ FWHM. The mapped area is 1.8 by $1 . ' 8$, each fiber corresponds to a circle of 4 .'2 on the sky.

For each galaxy, spaxel by spaxel, we modeled and subtracted the stellar continuum before fitting the most important emission-lines with a Gaussian. We produced an atlas of maps of the most relevant emission-lines in the spectra: [O II] $\lambda 3727$, $\mathrm{H} \gamma, \mathrm{H} \beta$, and [O III] $\lambda 5007$, of the continuum, of the extinction $\left(A_{\mathrm{V}}\right)$, and the excitation $([\mathrm{O} \mathrm{III}] \lambda 5007 / \mathrm{H} \beta)$ ratios, as well as of the gas and the stellar kinematics. Integrated and nuclear spectroscopic properties (line fluxes, extinction coefficient, and metal abundances) were also derived. From this study, we highlight the following results:

- All galaxies show a regular morphology in the continuum, with outer elliptical or circular isophotes; the ionized gas displays a more complex pattern, with filamentary emission and the presence of bubbles and holes. Remarkable are NGC 4670, where the gas emission shows two large extensions north and southwest of the center, and III $\mathrm{Zw} 102$, where the two narrow features protruding almost symmetrically from the main body, and a knot north of the galaxy center, form part of a polar ring.

- The galaxies display different ionization patterns: in II Zw 33 and Mrk 314 the excitation ratio, [O III] $\lambda 5007 / \mathrm{H} \beta$ traces the SF regions, as expected in objects ionized by stars. NGC 4670 has a somewhat complex pattern, with [O III] $\lambda 5007 / \mathrm{H} \beta$ peaking in the central [H II] region and at the outer edge of the two large filaments. These three galaxies have excitation ratios higher than one. In Haro 1 and III $\mathrm{Zw} 102, \mathrm{H} \beta$ is stronger than [O III], and the excitation does not trace the SF regions, but has a minimum in the central galaxy regions (where the emission peaks) and increases toward the galaxy outskirts.

- All galaxies possess significant amounts of dust, with interstellar reddening values up to $A_{\mathrm{V}} \sim 1.0$.

- A variety of kinematical behaviors are present in the sample galaxies. II $\mathrm{Zw} 33$ shows little or no rotation, while in Haro 1 gas and stars both display the same overall regular rotation pattern. The kinematics of NGC 4670 is typical of a barred galaxy, with S-shaped isovelocity contours in the gaseous component; stars co-rotate with the gas, albeit with a flatter gradient and lower amplitude. Mrk 314 has an irregular rotation pattern, with a drop in velocity in its southern lobe. The most complex object is III Zw 102, with two kinematically independent systems: an inner disk, where gas and stars co-rotate, and a polar ring orthogonal to it.

Acknowledgements. L. M. Cairós acknowledges the Alexander von Humboldt Foundation. VIRUS-P has been made possible by a generous donation from the Cynthia and George Mitchell Foundation. This paper includes data taken at The McDonald Observatory of the University of Texas at Austin. We thank J. N. González-Pérez and J. Falcón Barroso for stimulating discussions and tips on the data processing and analysis. N. Caon is grateful for the hospitality of the Leibniz-Institut für Astrophysik. This research has made use of the NASA/IPAC Extragalactic Database (NED), which is operated by the Jet Propulsion Laboratory, Caltech, under contract with the National Aeronautics and Space Administration. This work has been funded by the Spanish Ministry of Science and Innovation (MCINN) under the collaboration "Estallidos" (grants HA2006-0032, AYA2007-67965-C03-01 and AYA2010-21887-C04-04). P. Papaderos is supported by Ciencia 2008 Contract, funded by FCT/MCTES (Portugal) and POPH/FSE (EC).

\section{References}

Aller, L. H. 1984, Astrophysics and Space Science Library, Vol. 112, Physics of thermal gaseous nebulae

Arp, H. 1966, ApJS, 14, 1

Bergvall, N. 2012, in Star Forming Dwarf Galaxies, eds. P. Papaderos, S. Recchi, \& G. Hensler, 175

Bergvall, N., \& Östlin, G. 2002, A\&A, 390, 891

Bertola, F. 1987, in Structure and Dynamics of Elliptical Galaxies, ed. P. T. de Zeeuw, IAU Symp., 127, 135

Blanc, G. A., Heiderman, A., Gebhardt, K., Evans, N. J., \& Adams, J. 2009, ApJ, 704,842

Burstein, D., \& Heiles, C. 1982, AJ, 87, 1165

Cairós, L. M., Caon, N., Vílchez, J. M., González-Pérez, J. N., \& Muñoz-Tuñón, C. 2001a, ApJS, 136, 393

Cairós, L. M., Vílchez, J. M., González Pérez, J. N., Iglesias-Páramo, J., \& Caon, N. 2001b, ApJS, 133, 321

Cairós, L. M., Caon, N., García-Lorenzo, B., Vílchez, J. M., \& Muñoz-Tuñón, C. 2002, ApJ, 577, 164

Cairós, L. M., Caon, N., Papaderos, P., et al. 2003, ApJ, 593, 312

Cairós, L. M., Caon, N., García-Lorenzo, B., et al. 2007, ApJ, 669, 251

Cairós, L. M., Caon, N., Papaderos, P., et al. 2009a, ApJ, 707, 1676

Cairós, L. M., Caon, N., Zurita, C., et al. 2009b, A\&A, 507, 1291

Cairós, L. M., Caon, N., Zurita, C., et al. 2010, A\&A, 520, A90

Caon, N., Cairós, L. M., Aguerri, J. A. L., \& Muñoz-Tuñón, C. 2005, ApJS, 157, 218

Cappellari, M., \& Emsellem, E. 2004, PASP, 116, 138

de Vaucouleurs, G., de Vaucouleurs, A., \& Corwin, J. R. 1976, in Second reference catalogue of bright galaxies (Austin: University of Texas Press)

de Vaucouleurs, G., de Vaucouleurs, A., Corwin, Jr., H. G., et al. 1991, Third Reference Catalogue of Bright Galaxies

Deeg, H., Duric, N., \& Brinks, E. 1997, A\&A, 323, 323

Demoulin, M.-H. 1969, ApJ, 157, 69

Denicoló, G., Terlevich, R., \& Terlevich, E. 2002, MNRAS, 330, 69

Dopita, M. A., \& Sutherland, R. S. 1995, ApJ, 455, 468

Doublier, V., Comte, G., Petrosian, A., Surace, C., \& Turatto, M. 1997, A\&AS, 124,405

Doublier, V., Caulet, A., \& Comte, G. 1999, A\&AS, 138, 213

Fanelli, M. N., O'Connell, R. W., \& Thuan, T. X. 1988, ApJ, 334, 665

García-Lorenzo, B., Cairós, L. M., Caon, N., Monreal-Ibero, A., \& Kehrig, C. 2008, ApJ, 677, 201

Gil de Paz, A., \& Madore, B. F. 2005, ApJS, 156, 345 
Gil de Paz, A., Madore, B. F., \& Pevunova, O. 2003, ApJS, 147, 29

Gordon, D., \& Gottesman, S. T. 1981, AJ, 86, 161

Grimes, J. P., Heckman, T., Aloisi, A., et al. 2009, ApJS, 181, 272

Guseva, N. G., Papaderos, P., Izotov, Y. I., et al. 2003a, A\&A, 407, 75

Guseva, N. G., Papaderos, P., Izotov, Y. I., et al. 2003b, A\&A, 407, 91

Guseva, N. G., Papaderos, P., Izotov, Y. I., et al. 2003c, A\&A, 407, 105

Hawarden, T. G., Longmore, A. J., Tritton, S. B., Elson, R. A. W., \& Corwin, Jr., H. G. 1981, MNRAS, 196, 747

Hill, G. J., MacQueen, P. J., Tufts, J. R., et al. 2006, in SPIE Conf. Ser., 6269

Hill, G. J., MacQueen, P. J., Adams, J., et al. 2007, in BAAS, 39, AAS Meeting Abstracts, 747

Hill, G. J., MacQueen, P. J., Smith, M. P., et al. 2008, in SPIE Conf. Ser., 7014

Hunter, D. A., \& Elmegreen, B. G. 2004, AJ, 128, 2170

Hunter, D. A., van Woerden, H., \& Gallagher, III, J. S. 1994, ApJS, 91, 79

Izotov, Y. I., Schaerer, D., Blecha, A., et al. 2006a, A\&A, 459, 71

Izotov, Y. I., Stasińska, G., Meynet, G., Guseva, N. G., \& Thuan, T. X. 2006b, A\&A, 448, 955

Izotov, Y. I., Guseva, N. G., Fricke, K. J., et al. 2010, A\&A, 517, A90

James, B. L., Tsamis, Y. G., Barlow, M. J., et al. 2009, MNRAS, 398, 2

Klein, U., Wielebinski, R., \& Thuan, T. X. 1984, A\&A, 141, 241

Kniazev, A. Y., Pustilnik, S. A., Grebel, E. K., Lee, H., \& Pramskij, A. G. 2004, ApJS, 153, 429

Kunth, D., \& Östlin, G. 2000, A\&ARv, 10, 1

Li, J. G., Seaquist, E. R., Wrobel, J. M., Wang, Z., \& Sage, L. J. 1993, ApJ, 413 , 150

Loose, H.-H., \& Thuan, T. X. 1986, in Star-forming Dwarf Galaxies and Related Objects, 73

Lynds, B. T., \& Furenlid, I. 1973, ApJ, 186, 445

Marlowe, A. T., Meurer, G. R., Heckman, T. M., \& Schommer, R. 1997, ApJS, 112,285

Mas-Hesse, J. M., \& Kunth, D. 1999, A\&A, 349, 765

Mas-Hesse, J. M., Kunth, D., Tenorio-Tagle, G., et al. 2003, ApJ, 598, 858

Mazzarella, J. M., \& Boroson, T. A. 1993, ApJS, 85, 27

McCall, M. L., Rybski, P. M., \& Shields, G. A. 1985, ApJS, 57, 1

Méndez, D. I., Cairós, L. M., Esteban, C., \& Vílchez, J. M. 1999, AJ, 117, 1688

Moiseev, A. V. 2008, Astrophys. Bull., 63, 201

Moustakas, J., \& Kennicutt, Jr., R. C. 2006, ApJS, 164, 81

Murphy, J. D., Gebhardt, K., \& Adams, J. J. 2011, ApJ, 729, 129
Noeske, K. G., Papaderos, P., Cairós, L. M., \& Fricke, K. J. 2005, A\&A, 429, 115

Osterbrock, D. E. \& Ferland, G. J. 2006, Astrophysics of gaseous nebulae and active galactic nuclei, 2nd edn. (Sausalito, CA: University Science Books)

Papaderos, P., Loose, H.-H., Thuan, T. X., \& Fricke, K. J. 1996, A\&AS, 120, 207

Papaderos, P., Izotov, Y. I., Fricke, K. J., Thuan, T. X., \& Guseva, N. G. 1998, A\&A, 338, 43

Papaderos, P., Guseva, N. G., Izotov, Y. I., \& Fricke, K. J. 2008, A\&A, 491, 113

Paturel, G., Petit, C., Prugniel, P., et al. 2003, A\&A, 412, 45

Peterson, C. J., Thonnard, N., Rubin, V. C., \& Ford, Jr., W. K. 1978, ApJ, 219, 31

Pilyugin, L. S. 2000, A\&A, 362, 325

Pilyugin, L. S. 2001, A\&A, 369, 594

Pilyugin, L. S., \& Thuan, T. X. 2005, ApJ, 631, 231

Renka, L. L., \& Cline, A. K. 1984, Rocky Mountain J. Math., 14, 223

Sánchez-Blázquez, P., Peletier, R. F., Jiménez-Vicente, J., et al. 2006, MNRAS, 371,703

Schaerer, D., Contini, T., \& Pindao, M. 1999, A\&AS, 136, 35

Schlafly, E. F., \& Finkbeiner, D. P. 2011, ApJ, 737, 103

Schlegel, D. J., Finkbeiner, D. P., \& Davis, M. 1998, ApJ, 500, 525

Schneider, S. E., \& Salpeter, E. E. 1992, ApJ, 385, 32

Searle, L. 1971, ApJ, 168, 327

Shalyapina, L. V., Moiseev, A. V., Yakovleva, V. A., Hagen-Thorn, V. A., \& Barsunova, O. Y. 2004, Astron. Lett., 30, 583

Shi, F., Kong, X., Li, C., \& Cheng, F. Z. 2005, A\&A, 437, 849

Smith, H. E. 1975, ApJ, 199, 591

Stasińska, G. 2009, What can emission lines tell us?, ed. J. Cepa, 1

Thuan, T. X., \& Martin, G. E. 1981, ApJ, 247, 823

Vacca, W. D., \& Conti, P. S. 1992, ApJ, 401, 543

van Driel, W., Arnaboldi, M., Combes, F., \& Sparke, L. S. 2000, A\&AS, 141, 385

Vanzi, L., Cresci, G., Telles, E., \& Melnick, J. 2008, A\&A, 486, 393

Vorontsov-Velyaminov, B. A. 1959, in Atlas and catalog of interacting galaxies Walter, F., Brinks, E., Duric, N., \& Klein, U. 1997, AJ, 113, 2031

Whitmore, B. C., Lucas, R. A., McElroy, D. B., et al. 1990, AJ, 100, 1489

Yasuda, N., Fujisawa, K., Sofue, Y., et al. 1992, PASJ, 44, 1 